ESAIM: M2AN 55 (2021) S677-S704

https://doi.org/10.1051/m2an/2020048
ESAIM: Mathematical Modelling and Numerical Analysis

www.esaim-m2an.org

\title{
GENERAL POLYTOPAL $H$ (div)-CONFORMAL FINITE ELEMENTS AND THEIR DISCRETISATION SPACES
}

\author{
RÉmi Abgrall, Élise Le MÉlÉdo* And Philipp Öffner
}

\begin{abstract}
We present a class of discretisation spaces and $H$ (div)-conformal elements that can be built on any polytope. Bridging the flexibility of the Virtual Element spaces towards the element's shape with the divergence properties of the Raviart-Thomas elements on the boundaries, the designed frameworks offer a wide range of $H$ (div)-conformal discretisations. As those elements are set up through degrees of freedom, their definitions are easily amenable to the properties the approximated quantities are wished to fulfil. Furthermore, we show that one straightforward restriction of this general setting share its properties with the classical Raviart-Thomas elements at each interface, for any order and any polytopal shape. Then, to close the introduction of those new elements by an example, we investigate the shape of the basis functions corresponding to particular elements in the two dimensional case.
\end{abstract}

Mathematics Subject Classification. 65N30.

Received October 17, 2019. Accepted July 18, 2020.

\section{INTRODUCTION}

There has been recently a lot of activity in the design of methods able to deal with polygonal meshes. In the case of elliptic PDEs, one can mention $[3,5,11,13,20]$. One may also mention $[6,21,27]$ for fluid problems, both for compressible and incompressible flow. These are very partial lists only. In the case of hyperbolic problems, the flexibility of the discontinuous Galerkin method also enables, a priori, to deal with polygonal meshes, see [10], one of the very first papers after the seminal works of Reeds and Hill [25] and Lesaint-Raviart [19]. There are several reasons for this interest of the scientific community: it allows more flexibility in the geometry description and facilitate mesh adaptation, and several other reasons such as the status of hanging nodes.

There are many variants of the discontinuous Galerkin method, and one family of algorithms that has received a lot of attention recently is the so-called Flux Reconstruction methods [18, 23, 28].

The classical simplicial Flux Reconstruction approach involves a point-wise approximation of the flux in a finite-differece framework, modified by a term living only on the boundary in order to retrieve stability and local conservation. Up to some involved modifications, this method can be adapted to quadrangles and hexaheda. In any of those cases, the Flux Reconstruction technique can be rewritten as a Galerkin method applied to a perturbed flux, perturbation guaranteeing the local conservation and stability. The perturbation term is determined by a lifting technique involving a Raviart-Thomas [24] polynomial.

Keywords and phrases. H(div)-conformity, finite elements, Raviart-Thomas elements, polytopal elements.

1 Institute of Mathematics, University of Zurich, 8057 Zurich, Switzerland.

${ }^{*}$ Corresponding author: elise.lemeledo@math.uzh.ch 
Going further and using the Residual Distribution framework, we have shown in [1] how to construct schemes analogous to Flux Reconstruction for arbitrary polytopes, convex or non-convex. By using an entropy correction term, this method benefits from a non-linear entropy stability property.

In this paper, our primary motivation is to construct a Raviart-Thomas like approximation, so that we can reinterpret the correction term we introduce in [1] exactly as it is done for simplices in [28]. We are also interested in providing new $H(\mathrm{div})$-conformal discretisation spaces on arbitrary polytopes which we believe has its interest by itself.

The theory of $H$ (div)-conformal element has already been studied by Raviart and Thomas [24] and later generalized by Nédélec [22] or Brezzi et al. in the context of mixed finite element method [7]. More recently, a mixed Petrov-Galerkin scheme using Raviart-Thomas elements has also been investigated in [16]. However, up to the authors knowledge, those elements are limited to simplicial and quadrangular shapes.

Several attempts to use general polygons have been made $[9,17,26]$, but they usually make use of generalized barycentric coordinates and are delicate to handle in distorted non-convex elements. A first polygonal $H$ (div)conformal element has been proposed in [14] using gradient reconstruction and pyramidal sub-meshes tessellation. However, their construction requires some shape regularity within the mesh and the parallel with Raviart-Thomas spaces is limited to the lowest order space. Another approach using stabilisation techniques has been later investigated in [15], where if more flexibility on the element's shape has been achieved the parallel with the Raviart-Thomas elements is still limited to lowest-order simplicial shapes.

Some other approaches as Virtual Elements Method [11] introduced approximation spaces based on Poisson's solutions. Although more flexible towards the element's shape, those are scalar and not $H$ (div)-conformal. A first promising shape-flexible $H$ (div)-conformal discretisation has been recently proposed in [12], where the conformity property is enforced directly at the level of the boundaries normal components. As it therefore leaves on each boundary face only a scalar representation of the normal component, the boundary setting may appear quite restrictive, especially in applications for which a direct component-wise characterisation or tangential information is suitable. Therefore, its usage may be delicate when one targets a discretisation enhancing the representation of boundary's quantities. We rather focus on creating spaces enhancing a boundary characterisation of the discretised quantity itself, that links to the setting presented in [11]. The quantity of information available on an element is maximized at the boundary, while the $H(\operatorname{div})$-conformity is preserved.

In this paper, we propose a construction enhancing a boundary characterisation, that inherits the interface properties of the Raviart-Thomas elements and benefits from the shape flexibility of the Virtual Element discretisation. Moreover, rather than defining a basis on which the correction functions can be decomposed, this new setting offers a new element class that can be used as such in the construction of further numerical schemes. Note also that in [1], we do not need the explicit construction of this new elements, we only need the knowledge of degrees of freedom. In that sense, this construction is also in the spirit of VEM.

After briefly recalling the key ideas of the Raviart-Thomas elements, we introduce our new class of discretisation spaces. In a second time, we detail a possible definition of $H(\operatorname{div})$-conformal element to finally test them through the behaviour of their corresponding basis functions in the numerical results. For the sake of readability, proofs and further element examples will be given in the appendix. For interested readers, more details on the construction can be found in the extended technical report [2] and an application of those elements can be found in [1].

\section{NOTATIONS}

Throughout the paper, our notation will be the following:

\section{Geometrical notations}

- $x$ : spatial variable $x=\left(x_{1}, \ldots, x_{d}\right)^{T} \in \mathbb{R}^{d}$.

- $K$ : polytopal shape with interior $K$ and boundary $\partial K$.

- $\mathfrak{n}$ : number of faces of the polytope $K$.

- $f$ : generic face $f$ of the boundary $\partial K$. 
- $n$ : generic normal to a generic face $f$.

$-f_{j}: j$ th face of the boundary $\partial K$, where $j \in \llbracket 1, n \rrbracket$.

- $\partial_{j} K$ : hyper-face of $f \in \partial K$ for which the variable $x_{j}$ is fixed.

\section{Monomials and polynomial spaces}

- $\alpha$ : multi-index $\alpha=\left(\alpha_{1}, \ldots, \alpha_{d}\right)$ defining the monomial $x^{\alpha}=x_{1}^{\alpha_{1}} \ldots x_{d}^{\alpha_{d}}$.

- $\mathbb{Q}_{k}$ : space of polynomials of degree $\max \left(\alpha_{i}\right)$ is at most $k$, of dimension $(k+1)^{d}$.

- $\mathbb{P}_{k}$ : space of polynomials of degree $\sum_{i=1}^{d} \alpha_{i} \leq k$, of dimension $\left(\begin{array}{c}k+d \\ k\end{array}\right)$.

- $\mathbb{Q}_{[k]}$ : space of polynomials of degree $\max \left(\alpha_{i}\right)=k$.

- $\mathbb{P}_{[k]}$ : space of polynomials of degree $\sum_{i=1}^{i} \alpha_{i}=k$.

- $\mathbb{P}_{k_{1}, k_{2}, \ldots, k_{d}}$ : space of polynomials with degree in $x_{i} \leq k_{i}$.

- $\mathbb{P}_{k_{1}, \ldots,\left[k_{i}\right], \ldots, k_{d}}$ : space of polynomials with degree $k_{i}$ in $x_{i}$.

$-\mathbb{Q}_{-1}=\mathbb{P}_{-1}=\{0\}$.

For example, when considering an element in $2 \mathrm{D}$, a face is one-dimensional and can be parametrised as $x_{2}=a x_{1}+b$ for some constant $a$ and $b$. The space $\partial_{1} K$ is then the hyper-face of the line for which the variable $x_{1}$ is fixed, and $\mathbb{Q}_{k}\left(\partial_{1} K\right)$ is reduced to the space of constants. In $3 \mathrm{D}, \partial_{1} K$ would reduce to the line driven by $x_{2}$ and $\partial_{2} K$ to the line driven by $x_{1}$. There, $\mathbb{Q}_{k}\left(\partial_{1} K\right)$ would be the space of polynomials of degree $k$ whose monomials are only involving the terms $x_{2}$.

\section{Functional spaces}

- $\mathcal{R}_{k}(\partial K)=\left\{p \in L^{2}(K),\left.p\right|_{f_{i}} \in \mathbb{P}_{k}\left(f_{i}\right)\right.$ for every face $\left.f_{i} \in \partial K\right\}$.

- $H(\operatorname{div}, K)=\left\{u \in\left(L^{2}(K)\right)^{d}, \operatorname{div} u \in L^{2}(K)\right\}$.

\section{Operators}

- $\mathrm{X}_{i=1}^{d}$ : Cartesian product: $\mathbf{X}_{i=1}^{d}\left(x_{i}\right)=x=\left(x_{1}, \ldots, x_{d}\right)$.

- $\left\{\zeta_{i}\right\}_{i}$ : set containing the $d$ cyclic permutations of $\{k+1, \underbrace{k, \ldots, k}_{d-1 \text { times }}\}$.

- $\mathbb{1}$ : indicator function.

- o: Hadamard product.

\section{Classical Raviart-Thomas elements}

The spirit of the Raviart-Thomas elements is to work in a vectorial polynomial discretisation subspace of $H(\operatorname{div}, K)$ in which the functions are characterised separately on the boundary and within the elements. Doing so, the enforcement of the $H($ div)-conformity can be done at the interfaces by a specific choice of moment-based degrees of freedom acting only on the boundaries. As formalised by Nédelec [22], its definition on any simplicial reference shape $K$ contained in $\mathbb{R}^{d}$ reads

$$
\operatorname{RT}_{k}(K)=\left(\mathbb{P}_{k}(K)\right)^{d} \oplus x \mathbb{P}_{[k]}(K) .
$$

There, any element $p \in \mathrm{RT}_{k}(K) \subset\left(\mathbb{P}_{k+1}(K)\right)^{d}$ writes under the form

$$
p=\left(\begin{array}{c}
p_{1}+x_{1} q \\
p_{2}+x_{2} q \\
\vdots \\
p_{d}+x_{d} q
\end{array}\right):=\underset{i=1}{\mathbb{X}}\left(p_{i}+x_{i} q\right)=\underset{i=1}{\mathrm{X}} p_{i}+\underset{i=1}{\mathrm{X}} x_{i} q
$$

for some $p_{i} \in \mathbb{P}_{k}(K), i \in \llbracket 1, d \rrbracket$ and $q \in \mathbb{P}_{[k]}(K)$. Up to some straightforward computations, the dimension of $\mathrm{RT}_{k}(K)$ can be formulated as

$$
\operatorname{dim} \mathrm{RT}_{k}(K)=\operatorname{dim}\left(\mathbb{P}_{k-1}(K)^{d}\right)+(d+1) \operatorname{dim} \mathbb{P}_{k}(f) .
$$


Therefore, the definition of the element is done by setting internal and normal moments projecting respectively on the spaces $\mathbb{P}_{k-1}(K)^{d}$ and $\mathcal{R}_{k}(\partial K)$.

Definition 2.1 (Degrees of freedom). Any $q \in \mathrm{RT}_{k}(K)$ is determined by

$$
\begin{array}{lll}
\text { Normal moments: } & q \longmapsto \int_{\partial K} q \cdot n p_{k} \mathrm{~d} \gamma(x), \quad \forall p_{k} \in \mathcal{R}_{k}(\partial K), \\
\text { Internal moments: } & q \longmapsto \int_{K} q \cdot p_{k-1} \mathrm{~d} x, \quad \forall p_{k-1} \in\left(\mathbb{P}_{k-1}(K)\right)^{d},
\end{array}
$$

where $\mathrm{d} \gamma$ represents the Lebesgue measure on the faces.

The basis functions of $\mathrm{RT}_{k}(K)$ that are dual to those degrees of freedom verify

$$
\left.q \cdot n\right|_{\partial K} \in \mathbb{P}_{k}(\partial K) \quad \text { or }\left.\quad q \cdot n\right|_{\partial K} \equiv 0
$$

and are classified respectively as normal and internal basis functions. One can observe the $H$ (div)-conformity property: it reduces here to the continuity of the normal component across the boundary. Further divergence properties also hold directly by the nature of the approximation space [24], being a subspace of $H($ div, $K$ ).

Property 2.2 (Divergence properties). For any $q \in \mathrm{RT}_{k}(K)$, it holds:

$$
\left\{\begin{array}{l}
\operatorname{div} q \in \mathbb{P}_{k}(K) \\
\left.q \cdot n\right|_{\partial K} \in \mathcal{R}_{k}(\partial K) .
\end{array}\right.
$$

However, as the relation $(d+1) \operatorname{dim} \mathbb{P}_{k}(f)=\operatorname{dim} \mathcal{R}_{k}(\partial K)$ is only valid when the number of edges is $d+1$, this definition is very specific to the simplicial case. Therefore, when going to quads, the definition is changed by modifying the meaning of the polynomial degree, i.e. using $\mathbb{Q}_{k}$ spaces instead of $\mathbb{P}_{k}$ spaces. The $\mathrm{RT}_{k}(K)$ space then reads

$$
\mathrm{RT}_{k}(K)=\left(\mathbb{Q}_{k}(K)\right)^{d}+x \mathbb{Q}_{[k]}(K)={\underset{i=1}{d}}_{\mathbb{P}_{\zeta_{i}(k+1, k, \ldots, k)}}
$$

and benefits from a dimensional split similar to (2.3);

$$
\operatorname{dim} \mathrm{RT}_{k}(K)=2 d \operatorname{dim} \mathbb{Q}_{k}(f)+\operatorname{dim} \underset{i=1}{\mathrm{X}} \mathbb{P}_{\zeta_{i}(\{k-1, k, \ldots, k\})}(K) .
$$

A definition of degrees of freedom analogous to (2.4) can then be set up. However, this extension is very specific to quads and cannot be adapted to offer a discretisation framework for arbitrary polytopes (see Abgrall et al. [2] for details).

\section{A FRAMEWORK FOR ARBITRARY POLYTOPES}

In order to build a unifying discretisation framework, we have to define spaces $\mathbb{H}_{k}(K)$ that fulfil the following property:

Requirement 3.1 (Requirements on the discretisation space). The space $\mathbb{H}_{k}(K)$ is a finite dimensional vectorial subspace of $H(\operatorname{div}, K)$ whose dimension adapts to both the number of the polygon's faces and the discretisation order.

In addition, to be able to endow $H(\operatorname{div}, K)$-conformal elements $E_{k}(K)$ through definitions of degrees of freedom, we further ask the Requirement 3.2.

Requirement 3.2 (Requirements on the elements).

(1) For any space $\mathbb{H}_{k}(K)$, there exists a unisolvent set of degrees of freedom $\{\sigma\}$ that can be split into internal and normal subsets so that both the number of internal degrees of freedom and of the normal degrees of freedom per face do not depend on the shape of $K$. 
(2) The number of internal and normal degrees of freedom both increase strictly monotonically with the discretisation order.

Lastly, to ensure the existence of a split into internal and normal subsets of degrees of freedom that matches the classification (2.5) at the level of the dual basis functions, the feasibility of the Requirement 3.3 in $\mathbb{H}_{k}(K)$ is also needed.

Requirement 3.3 (Requirement on the basis functions). For any polytope $K$, the internal basis functions vanish on every face of the element.

One may possibly ask for one further requirement ensuring a parallel with the Raviart-Thomas setting from the lowest order on.

Requirement 3.4 (Optional requirement on the basis functions). The lowest order element has no internal degrees of freedom.

\subsection{A class of admissible approximation spaces.}

\section{Construction of spaces of discretisation}

In order to design a subspace of $H(\operatorname{div}, K)$ that satisfies Requirement 3.1 we are led to define a space $\mathbb{H}_{k}(K)$ with the same architecture as the classical Raviart-Thomas space. Thus, we look for spaces in the form $\mathbb{H}_{k}(K)=\left(A_{k}\right)^{d}+x B_{k}$ for two given functional sets $A_{k}$ and $B_{k}$. In order to design those two sets, we start by observing that the use of polynomial spaces is excluded by the Requirement 3.3, being required for any number of edges. Therefore, we consider the spaces $A_{k}$ and $B_{k}$ based on solutions to Poisson's problems as in the context of the VEM method [11]. There, a way to allow the existence in $\mathbb{H}_{k}(K)$ of smooth internal basis functions is to use the set of solutions to the boundary problems $\left\{\left.u\right|_{\partial K}=0, \Delta u=p_{k}\right\}$ for any $p_{k}$ belonging to $\mathbb{Q}_{m}(K), m \in \mathbb{N} \cup\{-1\}$.

In addition, as the $H(\operatorname{div}, K)$-conformity will be enforced by normal quantities that are tested only on the boundaries, we also consider the set of Poisson's problems $\left\{\left.u\right|_{\partial K}=p_{k} \mathbb{1}_{f}, \Delta u=0\right\}$ defined from polynomial boundary functions $p_{k} \in \mathbb{Q}_{l}(f), l \in \mathbb{N} \cup\{-1\}$ for each face $f$ of $\partial K$. Thus, seeing the boundary $\partial K$ face-wise, we define the set

$$
\mathcal{H}_{k}(\partial K)=\left\{\left.u\right|_{\partial K} \in L^{2}(\partial K),\left.u\right|_{f} \in \mathbb{Q}_{k}(f), \forall f \in \partial K\right\}
$$

and build the space $\mathbb{H}_{k}(K)$, for integers $l_{1}, l_{2}, m_{1}$ and $m_{2}$, as follows.

Definition $3.5\left(\mathbb{H}_{k}(K)\right.$ space).

$$
\begin{aligned}
\mathbb{H}_{k}(K)= & \left\{u \in H^{1}(K),\left.u\right|_{\partial K} \in \mathcal{H}_{l_{1}}(\partial K), \Delta u \in \mathbb{Q}_{m_{1}}(K)\right\}^{d} \\
& +x\left\{u \in H^{1}(K),\left.u\right|_{\partial K} \in \mathcal{H}_{l_{2}}(\partial K), \Delta u \in \mathbb{Q}_{\left[m_{2}\right]}(K)\right\} .
\end{aligned}
$$

The choice of $l_{1}, l_{2}, m_{1}$ and $m_{2}$ is related to $k$ and will be discussed below.

\section{Remark 3.6.}

- The two subspaces in the definitions of $\mathbb{H}_{k}(K)$ are in direct sum whenever $l_{1} \leq 0$.

- The presented space is based on polynomial spaces $\mathbb{Q}_{k}(K)$ rather than $\mathbb{P}_{k}(K)$ for the sake of consistency with the definition of the Raviart-Thomas space built on quads. This is also a more natural choice when considering mappings to elements of reference, as the monomials involved in the transformations a more coherent with a $\mathbb{Q}_{k}(K)$ based discretisation (especially for the lower order discretisation where the monomial $x y$ is not part of $\mathbb{P}_{1}(K)$ but already belongs to $\mathbb{Q}_{1}(K)$ ). 
Properties of $\mathbb{H}_{k}(K)$ spaces

The space $\mathbb{H}_{k}(K)$ is constructed from four independent blocks whose definitions are driven by the independent coefficients $l_{1}, l_{2}, m_{1}$ and $m_{2}$. The couple $\left(m_{1}, m_{2}\right)$ drives the discretisation quality exclusively within the cell while $\left(l_{1}, l_{2}\right)$ takes care only of the boundary. Thus, the separation between internal and normal basis functions is natural. Furthermore, the Property 3.7 holds, emphasising that the $H(\operatorname{div}, K)$-conformity is ensured by the definition of $\mathcal{H}_{k}(\partial K)$, while the inner smoothness is provided through the Laplacian.

Proposition 3.7. For any function $q$ belonging to any space $\mathbb{H}_{k}(K)$, it holds:

$$
\left.q \cdot n\right|_{\partial K} \in \mathcal{H}_{\max \left\{l_{1}, l_{2}\right\}}(\partial K) \text { and } \operatorname{div} q \in L^{2}(K) .
$$

It comes the following inclusion allowing $H$ (div)-conformity.

Corollary 3.8. For any couples $\left(l_{1}, l_{2}\right)$ and $\left(m_{1}, m_{2}\right)$,

$$
\mathbb{H}_{k}(K) \subset H(\operatorname{div}, K) \text {. }
$$

When selecting $l_{1} \leq 0$, those spaces are of dimension

$$
\operatorname{dim} \mathbb{H}_{k}(K)=\mathfrak{n}\left(d\left(l_{1}+1\right)^{d-1}+\left(l_{2}+1\right)^{d-1}\right)+\left(d\left(m_{1}+1\right)^{d}+\left(m_{2}+1\right)^{d}-m_{2}^{d}\right),
$$

making their structure a priori suitable to be used as discretisation spaces endowing $H(\operatorname{div}, K)$-conformal elements.

Example 3.9. When $K$ is a two-dimensional simplex and when $l_{1}, l_{2}$ are chosen as $\left(l_{1}, l_{2}\right)=(-1, k)$, the discretisation quality of the normal component matches the one of the Raviart-Thomas setting.

Admissibility of the spaces for building $H$ (div)-conformal elements

In order to define elements in the spirit of Raviart-Thomas, we need to set $\left(d\left(l_{1}+1\right)^{d-1}+\left(l_{2}+1\right)^{d-1}\right)$ normal degrees of freedom per face and $d\left(m_{1}+1\right)^{d}+\left(m_{2}+1\right)^{d}-m_{2}^{d}$ internal degrees of freedom. While this splitting does not impact the set of admissible coefficients $\left(m_{1}, m_{2}\right)$, it reduces the range of coefficients $\left(l_{1}, l_{2}\right)$ that can be used. Indeed, the space $\mathbb{H}_{k}(K)$ is constructed from four independent blocks providing two distinct discretisations: on the boundary and within the element. Thus, when testing a function of $\mathbb{H}_{k}(K)$ through normal degrees of freedom, one can only retrieve the polynomial obtained from the two boundary conditions defining the sets $A_{k}$ and $B_{k}$. On each face, this polynomial is of the form $p=p_{k, A}+p_{k, B}$, where the function $\left.p_{k, A} \in\left(A_{k}\right)^{d}\right|_{f}$ reads

$$
p_{k, A}=\underset{j=1}{\underset{X}{X}}\left(\sum_{\left|\alpha_{i}\right| \leq l_{1}} a_{i j} x^{\alpha_{i}}\right)
$$

for a given set of multi-index $\left\{\alpha_{i}\right\}_{i}$ and coefficients $\left\{a_{i, j}\right\}_{i, j}$ depending on the coordinates $x_{j}$. The function $\left.p_{k, B} \in x B_{k}\right|_{f}$ reads however

$$
p_{k, B}=\underset{j=1}{\stackrel{d}{X}}\left(x_{j} \sum_{\left|\beta_{i}\right| \leq l_{2}} b_{i} x^{\beta_{i}}\right)
$$

for a given set of multi-indices $\left\{\beta_{i}\right\}_{i}$ and coefficients $\left\{b_{i}\right\}_{i}$ independent of the coordinates $x_{j}$. Therefore, denoting by $\left\{\xi_{j}\right\}_{j \in \llbracket 1, d \rrbracket}$ the coordinates permutation that allows to shift the lowest orders terms of $\left.x B_{k}\right|_{f}$ to $\left.\left(A_{k}\right)^{d}\right|_{f}$, $\left.p \in \mathbb{H}_{k}(K)\right|_{f}$ can be written as follows:

$$
\text { If } \begin{aligned}
l_{2} \geq l_{1}, \quad p & =\underset{j=1}{\underset{X}{X}}\left(\sum_{\left|\alpha_{i}\right| \leq l_{1}} a_{i j} x^{\alpha_{i}}\right)+\underset{j=1}{\underset{X}{X}}\left(x_{j} \sum_{\left|\beta_{i}\right| \leq l_{2}} b_{i} x^{\beta_{i}}\right) \\
& =\underset{j=1}{\underset{X}{X}}\left(\sum_{\substack{\left|\alpha_{i}\right| \leq l_{1} \\
\left|\alpha_{i}\right| \neq 0}}\left(a_{i j}+b_{\xi_{j}(i)}\right) x^{\alpha_{i}}+x_{j} \sum_{l_{1} \leq\left|\beta_{i}\right| \leq l_{2}} b_{i} x^{\beta_{i}}\right)+\underset{j=1}{\underset{X}{X}} a_{0 j} x_{j}^{0} .
\end{aligned}
$$




$$
\text { If } l_{1} \geq l_{2}+1, \quad p=\underset{j=1}{X}\left(\sum_{\substack{\left|\alpha_{i}\right| \leq l_{1} \\\left|\alpha_{i} \neq 0\right|}}\left(a_{i j}+b_{\xi_{j}(i)}\right) x^{\alpha_{i}}+a_{0 j} x_{j}^{0}\right) .
$$

The structure of those relations implies that the terms $a_{i j}$ and $b_{\xi_{j}(i)}$ are combined into a single coefficient and cannot be specified individually from further normal degrees of freedom. Indeed, the remaining freedom can only be seen inside the polytope, as a consequence of the boundary conditions on the Poisson's solutions in either $A_{k}$ or $x B_{k}$. To prevent any over-determination by the normal degrees of freedom in $\left.\mathbb{H}_{k}(K)\right|_{f}$, we therefore have to make sure that the dimension of the boundary part (3.7) and (3.8) of any function living in $\mathbb{H}_{k}(K)$ is larger than the number of wished normal degrees of freedom per face. By reading out the structure of (3.7) and (3.8) it comes

$$
\left.\operatorname{dim} \mathbb{H}_{k}(K)\right|_{f}= \begin{cases}\left(l_{2}+1\right)^{d-1} & \text { if } l_{1}=-1, \\ d\left(l_{1}+1\right)^{d-1}+\left(l_{2}+1\right)^{d-1}-l_{1}^{d-1} & \text { if } l_{2} \geq l_{1}, \\ d\left(l_{1}+1\right)^{d-1} & \text { otherwise }\end{cases}
$$

We thus restrict the admissible couples $\left(l_{1}, l_{2}\right)$ to those verifying the Admissibility conditions 3.10, preventing any over-determination.

Admissibility conditions 3.10 (Necessary condition for using conformal elements). If $\operatorname{dim} \mathcal{N}$ is the number of normal moments per face that we wish, and $\operatorname{dim} \mathbb{H}_{k}(K)_{\mid f}$ is the number of coefficient we can tune for the face $f$, we should have:

$$
\operatorname{dim} \mathcal{N} \leq \operatorname{dim} \mathbb{H}_{k}(K)_{\mid f} .
$$

In the case $l_{2} \geq l_{1}$, it reduces to:

$$
d\left(l_{1}+1\right)^{d-1}+\left(l_{2}+1\right)^{d-1} \leq d\left(l_{1}+1\right)^{d-1}+\left(l_{2}+1\right)^{d-1}-l_{1}^{d-1} \quad\left(\Leftrightarrow \quad l_{1}^{d-1} \leq 0\right)
$$

while otherwise it comes

$$
d\left(l_{1}+1\right)^{d-1}+\left(l_{2}+1\right)^{d-1} \leq d\left(l_{1}+1\right)^{d-1}
$$$$
\left(\Leftrightarrow \quad l_{2}=-1\right) .
$$

Regarding the internal characterisation, any couple of coefficients $\left(m_{1}, m_{2}\right)$ is allowed.

\section{Definition of series of spaces}

While fulfilling the above conditions, one can set a specific discretisation framework within which the spaces share a predefined structure. By example, defining the four coefficients $l_{1}, l_{2}, m_{1}$ and $m_{2}$ through affine relations of the type $l=a k+b$ for some index $k \in \mathbb{N}$, the range of discretisation qualities achievable within the framework is predetermined by a refinement sequence in each block, and the order of each space can be simply defined as the index $k$ generating each of the four coefficients. A typical working example is obtained by defining $m_{1}=m_{2}=k-1, l_{1}=0$ and $l_{2}=k$, leading to a series of discretisation spaces of order $k$. This case is specifically detailed in the Section 3.4.

\subsection{Definition of admissible elements}

Under the Admissibility condition 3.10, the spaces $\mathbb{H}_{k}(K)$ allow the construction of $H($ div)-conformal elements through the definition of normal degrees of freedom enforcing the conformity and internal ones preserving it. We propose here a possible construction of such sets.

\section{Definition of admissible normal degrees of freedom}

The role of the normal degrees of freedom is to determine vectorial polynomials on the boundaries and to enforce the $H$ (div)-conformity of the element. We define them as the normal component of the tested quantities projected against polynomials of $\mathbb{H}_{k}(K)_{\mid f}$. We focus on the following possibilities: 
Available types of degrees of freedom. For any $q \in \mathbb{H}_{k}(K)$, we define:

(1) The face integral of coordinate-wise components tested against projection polynomials p:

$$
q \mapsto \int_{f} q_{i} n_{i x} p \mathrm{~d} \gamma(x), \quad \forall p \in \mathbb{Q}_{\max \left\{l_{1}, l_{2}\right\}}(f) .
$$

(2) The face integral of a function in $\mathbb{H}_{k}(K)$ projected onto the face normal, and tested against polynomials:

$$
\begin{aligned}
q & \mapsto \int_{f} q \cdot n p \mathrm{~d} \gamma(x), \quad \forall p \in \mathbb{Q}_{\max \left\{l_{1}, l_{2}\right\}}(f), \\
q & \mapsto \int_{f} q \cdot n p \mathrm{~d} \gamma(x), \quad \forall p \in\left\{x_{i} p_{i}, p_{i} \in \mathbb{Q}_{\zeta_{i}\left(\left[l_{2}\right], l_{2}, \ldots, l_{2}\right)}(f), i \in \llbracket 1, d-1 \rrbracket\right\} .
\end{aligned}
$$

(3) The pointwise values of the discretised quantity tested against the face's normal:

$$
q \mapsto q\left(x_{i m}\right) \cdot n_{i} \text {, for sampling points }\left\{x_{i m}\right\}_{m} \text { on the face } f_{i} \text {. }
$$

Defining the normal degrees of freedom then reduces to choosing $d\left(l_{1}+1\right)^{d-1}+\left(l_{2}+1\right)^{d-1}$ of them among the possibilities (3.10) so that their set is unisolvent for $\left.\mathbb{H}_{k}(K)\right|_{f}$. To ensure this, preventing any under-determination is sufficient. Therefore, we need to avoid the selection of projectors that are linearly dependent, and pay attention to determining both global and coordinate-wise behaviours of any vector polynomial $q \in \mathbb{H}_{k}(K)_{\mid f}$.

Example 3.11. In two dimensions and for $l_{1}=l_{2}=0$, any $p \in \mathbb{H}_{k}(K)_{\mid f}$ reads

$$
q=\left(\begin{array}{l}
A \\
B
\end{array}\right)+C\left(\begin{array}{l}
x \\
y
\end{array}\right)
$$

for some constants $A, B$ and $C$. The characterisation of $q$ can be done by selecting two component-wise moments involving $A n_{i x}$ or $B n_{i y}$ tested against the constant polynomial $p=1$ and one global moment that tests $q \cdot n=C\left(n_{i x}+n_{i y}\right)+A n_{i x}+B n_{i y}$ against the polynomial $p=x$. One could also choose two global moments and one coordinate-wise.

In practice, the selection of degrees of freedom reduces to choosing the polynomials $p$ on which the function $q$ will be tested coordinate-wise. The other polynomials $p$ play the role of test functions for the global normal component $q \cdot n$. The unisolvence of the set is then ensured by the following admissibility conditions.

\section{Admissibility conditions 3.12.}

(1) The projection polynomials $p$ involved in (3.10a), (3.10b) and (3.10c), and all the forms (3.10d) that define the point values must be linearly independent.

(2) When using a coordinate-wise degree of freedom of the type (3.10a), polygonal shapes $K$ containing a face parallel to any axis are not allowed. The term $n_{i x}$ or $n_{i y}$ would indeed always vanish for some $i \in \llbracket 1, n \rrbracket$, thus not describing any function of $\mathbb{H}_{k}(K)_{\mid f}$.

Note. The second point of the admissibility conditions may seem unreasonable as it may prevent the use of some shapes for specific orientations. However, it is always possible to easily modify the incriminated moments element-wise or to select other moments that make the element robust with respect to rotation while still yielding $H$ (div)-conformity. See Abgrall et al. [2] for more details.

To help the construction of an element on $K$ through the selection of degrees of freedom among those fulfilling the Admissibility condition 3.12, we recall that the chosen set of degrees of freedom imposes the shape of the dual basis functions. We can therefore select the degrees of freedom depending on the wished properties of the basis functions.

More crucially, the selection of global and/or coordinate-wise normal degrees of freedom leads to the reclassification of some basis functions as internal ones. Indeed, as the face-wise normal component of any function $q$ in $\mathbb{H}_{k}(K)_{\mid f}$ is only of degree $\max \left\{l_{1}, l_{2}\right\}$, the term $\left.q \cdot n\right|_{f}$ requires only $\left(\max \left\{l_{1}, l_{2}\right\}+1\right)^{d-1}$ basis functions to be decomposed on. Therefore, up to $d\left(l_{1}+1\right)^{d-1}$ basis functions may see their global normal component 
vanishing on every face. Their coordinate-wise components will however not vanish, as they take care of the coordinate-wise behaviours that cannot be determined solely through the expression of $\left.q \cdot n\right|_{f}$.

Remark 3.13. Typically, the more global degrees of freedom are designed, the more the representation of $q \cdot n$ is completed globally. As a consequence, more basis functions have a vanishing normal component as they are forced to take care only of coordinate-wise behaviours, forcing them to be reclassified into internal basis function. The reverse scenario may also be considered.

To avoid this reclassification and allow a parallel with the Raviart-Thomas elements from the lowest order space, we ask the Requirement 3.4. This will be discussed in the Section 3.4.2.

An example of a possible definition of normal degrees of freedom

As an example, we detail one selection of normal degrees of freedom in the case $l_{2} \geq l_{1}$ where every function in $\left.\mathbb{H}_{k}(K)\right|_{f}$ is of the form (3.7). For interested readers, other possibilities are presented in the technical report [2].

Here, we select moments from the set (3.10) so that the elements of $\mathbb{H}_{k}(K)_{\mid f}$ are determined as much as possible by testing only their normal component. The remaining freedom is characterised by few coordinate-wise moments. We consider:

$$
\begin{array}{ll}
\sigma: q \mapsto \int_{f_{j}} q_{i} n_{x_{i}} x_{i}^{l_{1}+1} \mathrm{~d} \gamma(x), & \text { for all } j \in \llbracket 1, \mathfrak{n} \rrbracket \text { and all } i \in \llbracket 1, d \rrbracket, \\
\sigma: q \mapsto \int_{f_{j}} q \cdot n p_{k} \mathrm{~d} \gamma(x), & \text { for all } j \in \llbracket 1, \mathfrak{n} \rrbracket \text { and all } p_{k} \in \mathbb{Q}_{l_{2}}\left(f_{j}\right) \backslash \mathbb{Q}_{l_{1}}\left(f_{j}\right), \\
\sigma: q \mapsto \int_{f_{j}} q \cdot n x_{j} x_{j}^{l_{2}} \tilde{x} \mathrm{~d} \gamma(x), & \text { for all } j \in \llbracket 1, \mathfrak{n} \rrbracket \text { and any } \tilde{x} \in \mathbb{Q}_{l_{2}}\left(\partial_{j} K\right),
\end{array}
$$

where $\tilde{x} \in \mathbb{Q}_{l_{2}}\left(\mathbb{R}^{d-2}\right)$ is not involving the variable $x_{j}$ so that the moment (3.11c) has for integrands the second terms of the right hand side of (3.7) when $\left|\beta_{i}\right|=l_{2}$. Note that the set (3.11) is of dimension $d\left(l_{1}+1\right)^{d-1}+$ $\left(l_{2}+1\right)^{d-1}-\left(l_{1}+1\right)^{d-1}+(d-1)\left(l_{2}+1\right)^{d-2}$ though we require $d\left(l_{1}+1\right)^{d-1}+\left(l_{2}+1\right)^{d-1}$ moments. Thus, this configuration can only be used when $l_{1}$ and $l_{2}$ verify the feasibility condition:

$$
\left(l_{1}+1\right)^{d-1} \leq(d-1)\left(l_{2}+1\right)^{d-2},
$$

which is a reduction of the Admissibility conditions 3.10. In two dimensions the above relation reduces to an equality, and all the degrees of freedom presented in (3.11) are considered. In higher dimensions, a further selection from the set (3.11) is required. There, we consider the sets (3.11a) and (3.11b) fully and select any $\left(l_{1}+1\right)^{d-1}$ moments from $(3.11 \mathrm{c})$.

Definition 3.14. Any choice of $\left(l_{1}+1\right)^{d-1}$ moments among (3.11c) is denoted as the "configuration Ia". Associated with any admissible internal degrees of freedom, its unisolvence is given by the Lemma B.1.

Up to the additional coordinate-wise moments, the configuration Ia is close to the Raviart-Thomas setting. However, the scaling of the dual basis functions does not match the one of the Raviart-Thomas basis. In order to obtain a similar scaling, one should rather scale the above degrees of freedom with respect to each edge's length and orientation, or consider in place of the moments (3.11c) the point-wise values

$$
q \mapsto q\left(x_{i m}\right) \cdot n,
$$

where $i \in \llbracket 1, d \rrbracket, m \in \llbracket 1,\left(1+l_{1}\right)^{d-1} \rrbracket$ and $x_{i m}$ is any sampling point on the face $f_{i}$.

Definition 3.15. Any selection of $\left(l_{1}+1\right)^{d-1}$ degrees of freedom among the sets (3.11a), (3.11b) and (3.13) is labelled as the "configuration Ib". Associated with any admissible internal degrees of freedom, its unisolvence is given by the Lemma B.1.

Remark 3.16. As we assume in this example that $l_{2} \geq l_{1}$, the choice of $l_{1}$ is restricted to either $l_{1}=0$ or $l_{1}=-1$. Thus, in the Definitions 3.14 and 3.15 , one would only need to select respectively one or none degrees of freedom from the set (3.11c) or (3.13). 
Definition of admissible internal degrees of freedom

In order to define admissible internal degrees of freedom, we have to make sure that the corresponding internal basis functions vanish on every face. We therefore stick to the idea of Raviart-Thomas and define moment based degrees of freedom that read for any $q \in \mathbb{H}_{k}(K)$

$$
\sigma(q) \mapsto \int_{K} q \cdot p_{k} \mathrm{~d} x, \quad \text { for all } p_{k} \in \mathcal{P}(K)
$$

for some function space $\mathcal{P}(K)$ of dimension $\left(\left(m_{1}+1\right)^{d}+\left(m_{2}+1\right)^{d}\right)$. Considered as a test space, $\mathcal{P}(K)$ may simply gather polynomial functions used in the definition of the Poisson's problems generating $\mathbb{H}_{k}(K)$. The discretised quantities would then be determined through their polynomial projections. Another choice is to test against the set of Poisson's solutions to the problems $\left\{\Delta p_{k} \in \mathcal{P},\left.p_{k}\right|_{\partial K}=0\right\}$.

Using one or the other possibility for $\mathcal{P}(K)$, the unisolvence of the set of internal degrees of freedom in $\left.\mathbb{H}_{k}(K)\right|_{K}$ is ensured by the following admissibility conditions (see the proof B.1, Part 3):

\section{Admissibility conditions 3.17.}

(1) The polynomials $\left\{p_{l}\right\}_{l}$ generating $\mathcal{P}(K)$ are linearly independent.

(2) No polynomial $p_{l}$ is of degree larger than $\max \left\{m_{1}, m_{2}+1\right\}$.

Definition of the elements

Combining the two previous paragraphs with the definition of the space $\mathbb{H}_{k}(K), H(\operatorname{div}, K)$-conformal elements can be set up.

Proposition 3.18. Let $K$ be any polytope satisfying the second item of the Admissibility conditions 3.12 and $\mathbb{H}_{k}(K)$ be any admissible space built on it. Let also $\left\{\sigma_{N}\right\}$ be any selection of $d\left(l_{1}+1\right)^{d-1}+\left(l_{2}+1\right)^{d-1}$ degrees of freedom from the set (3.10) fulfilling the first item of the Admissibility conditions 3.12, and $\left\{\sigma_{I}\right\}$ the set of internal moments built through the expression (3.14) for any of the projection sets $\mathcal{P}(K)$ fulfilling the Admissibility conditions 3.17. Then, the set $\left\{\sigma_{N}\right\} \cup\left\{\sigma_{I}\right\}$ is unisolvent for $\mathbb{H}_{k}(K)$ and defines a $H(\operatorname{div}, K)$-conformal element.

This well-possessedness property is an immediate corollary of the following proposition, proven in the Appendix B.

Proposition 3.19. Let $q \in \mathbb{H}_{k}(K)$, and denote $\sigma_{N}(q)$ the $n$-tuple of normal degrees of freedom extracted from the set (3.10). If

$$
\sigma_{N}(q)=0 \text { and } \int_{K} q \cdot p_{k} \mathrm{~d} x=0 \quad \text { for all } p_{k} \in \mathcal{P}
$$

then $q=0$.

At this point, any admissible definition leads to $H(\operatorname{div}, K)$-conformal elements.

\subsection{Summary of the construction}

Let us summarize the spaces construction and the example of normal degrees of freedom that has been detailed above. To begin with, the class of discretisation spaces reads

$$
\begin{aligned}
\mathbb{H}_{k}(K)= & \left\{u \in H^{1}(K),\left.u\right|_{\partial K} \in \mathcal{H}_{l_{1}}(\partial K), \Delta u \in \mathbb{Q}_{m_{1}}(K)\right\}^{d} \\
& \oplus x\left\{u \in H^{1}(K),\left.u\right|_{\partial K} \in \mathcal{H}_{l_{2}}(\partial K), \Delta u \in \mathbb{Q}_{\left[m_{2}\right]}(K)\right\},
\end{aligned}
$$

with the convention that $\mathbb{Q}_{-1}=\{0\}$ and where the integers $l_{1}, l_{2}, m_{1}$ and $m_{2}$ verify

$$
m_{1}, m_{2}, l_{2} \geq-1 \quad \text { and } \quad-1 \leq l_{1} \leq 0
$$

So defined, it holds:

$-\operatorname{dim} \mathbb{H}_{k}(K)=\mathfrak{n}\left(d\left(l_{1}+1\right)^{d-1}+\left(l_{2}+1\right)^{d-1}\right)+\left(\left(m_{1}+1\right)^{d}+\left(m_{2}+1\right)^{d}-m_{2}^{2}\right)$

- For all $q \in \mathbb{H}_{k}(K),\left.q \cdot n\right|_{\partial K} \in \mathcal{H}_{\max \left\{l_{1}, l_{2}\right\}}(\partial K)$ and $\mathbb{H}_{k}(K) \subset H(\operatorname{div}, K)$. 
TABLE 1. Summary of the used degrees of freedom for the configurations Ia.

\begin{tabular}{llll}
\hline \hline Representation & Of low order & Of $\left.\left(A_{k} \cap B_{k}\right)\right|_{\partial K}$ & Of higher orders \\
\hline $\begin{array}{l}\text { Available when } \\
(d-1)\left(l_{2}+1\right)^{d-2}\end{array}$ & $\begin{array}{l}\text { Inherited from } \\
\text { the highest order rep- }\end{array}$ & $\int_{f_{j}} q_{i} n_{x_{i}} x_{i}^{l_{1}+1}$ & $\int_{f_{j}} q \cdot n p_{k}$ \\
$\geq\left(l_{1}+1\right)^{d-1}$ & & $\int_{\boldsymbol{f}_{j}} \boldsymbol{q} \cdot \boldsymbol{n} \boldsymbol{x}_{\boldsymbol{i}} \boldsymbol{x}_{\boldsymbol{i}}^{\boldsymbol{l}_{\boldsymbol{i}} \tilde{\boldsymbol{x}}}$ \\
$\begin{array}{l}\text { resentation } \\
\text { Select }\left(l_{1}+1\right)^{d-1}\end{array}$ & $\forall i \in \llbracket 1, d \rrbracket$, & \\
moments per face & $\forall j \in \llbracket 1, \mathfrak{n} \rrbracket$ & $\forall p_{k} \in \mathbb{Q}_{l_{2}}\left(f_{j}\right) \backslash$ \\
from the bold ones. & & $\mathbb{Q}_{l_{1}}\left(f_{j}\right)$, \\
& & $\forall \tilde{x} \in \mathbb{Q}_{l_{2}}\left(\partial_{i} K\right)$ \\
\hline
\end{tabular}

Thus, conformal elements can be defined through normal degrees of freedom enforcing the $H$ (div)-conformity and internal ones preserving it, provided that the polytope $K$ satisfies the two conditions

- The polytope $K$ has a reasonable aspect ratio, so that the Poisson problem (required by the definition of the underlying VEM spaces) is well posed.

- No face is parallel to any axis, ensuring the unisolvence of the presented degrees of freedom (when using component-wise degrees of freedom).

Note that when selecting component-wise degrees of freedom, the above condition on the orientation of the face with respect to the axis raises stability issues when dealing with element whose faces are almost parallel to the axis. This issue is easily avoidable by selecting at least a global degree of freedom involving the term $p \cdot n$ in the moment's integrand and changing the testing vector in the coordinate-wise degrees of freedom to any vector $v \neq n$.

Internal degrees of freedom. It is set

$$
\sigma: q \mapsto \int_{K} q \cdot p_{k} \mathrm{~d} x, \quad \forall p_{k} \in \mathcal{P}
$$

for any space $\mathcal{P}$ defined either as a polynomial space or as any subspace of Poisson's solutions, having for dimension $\left(m_{1}+1\right)^{d}+\left(m_{2}+1\right)^{d}-m_{2}^{d}$ and fulfilling the Assumption 3.17.

Normal degrees of freedom. Though in two dimensions the above setting is fixed and all the mentioned degrees of freedom have to be considered, in three dimensions the selection of degrees of freedom among the bold ones is a matter of taste, possibly directed by properties of the discretised quantities that are known a priori. Note also that one could project on any other polynomial basis rather than using projections over monomials (Table 1).

\subsection{Two examples in two dimensions}

We first detail an example of a discretisation framework contained in the previously presented setting for which a parallel with the Raviart-Thomas elements can be drawn from the order $k=1$ on. In a second time, we present an example of a reduced framework where a parallel with the Raviart-Thomas is achieved at any order.

\subsubsection{An example of a general setting}

We consider a series of discretisation spaces by indexing the coefficients $l_{1}=0, l_{2}=k, m_{1}=k-1$ and $m_{2}=k-1$ for any $k \in \mathbb{N}$, seen here as the space order. The space $\mathbb{H}_{k}(K)$ is then defined as

$$
\begin{aligned}
\mathbb{H}_{k}(K)= & \left\{u \in H^{1}(K),\left.u\right|_{\partial K} \in \mathcal{H}_{0}(\partial K), \Delta u \in \mathbb{Q}_{k-1}(K)\right\}^{2} \\
& \oplus x\left\{u \in H^{1}(K),\left.u\right|_{\partial K} \in \mathcal{H}_{k}(\partial K), \Delta u \in \mathbb{Q}_{[k-1]}(K)\right\} .
\end{aligned}
$$


TABLE 2. Definition of the degrees of freedom in the 2D case for the configuration Ia.

\begin{tabular}{llll}
\hline \hline Representation & Of low order & Of $\left.\left(A_{k} \cap B_{k}\right)\right|_{\partial K}$ & Of higher orders \\
\hline Moments & $\int_{f_{j}} q \cdot n$ & $\int_{f_{j}} q_{i} n_{x_{i}} x_{i}$ & $\int_{f_{j}} q \cdot n p_{k}$ \\
& $\forall j \in \llbracket 1, \mathfrak{n} \rrbracket$ & $\forall i \in \llbracket 1,2 \rrbracket, \forall j \in$ & $\forall j \in \llbracket 1, \mathfrak{n} \rrbracket$, \\
& & $\llbracket 1, \mathfrak{n} \rrbracket$ & $\forall p_{k} \in \mathbb{Q}_{k}\left(f_{j}\right) \backslash \mathbb{Q}_{0}\left(f_{j}\right)$
\end{tabular}

By a straightforward application of the previous section, it comes

$$
\operatorname{dim} \mathbb{H}_{k}(K)=\mathfrak{n}(k+3)+2 k(k+1)-\mathbb{1}_{k>0} .
$$

\section{Example of a two dimensional element}

The example of selected normal degrees of freedom defining the elements $E_{k}=\left(K, \mathbb{H}_{k}(K),\{\sigma\}\right)$ presented in the previous section then reduces to the expressions given in the Table 2. The internal degrees of freedom are set as

$$
\sigma(q) \mapsto \int_{K} q \cdot p_{k} \mathrm{~d} x, \quad \text { for all } p_{k} \in \mathcal{P}
$$

where $\mathcal{P}$ is chosen as the space

$$
\mathcal{P}=\mathbb{P}_{k, k-1} \times \mathbb{P}_{k-1, k} \backslash\left(\mathbb{P}_{[k],[k-1]} \times \mathbb{P}_{[k-1],[k]}\right) \cup\left\{(x, y)^{T} \mapsto\left(\begin{array}{c}
x^{k} y^{k-1} \\
x^{k-1} y^{k}
\end{array}\right)\right\} .
$$

Though the internal projection space is less refined than the one set on the edges, this is not bothersome as the impact of the divergence within the cell is less dramatic. Note also that in practice, for defining the projections (3.18) one can work with any basis of $\mathcal{P}$.

Link to another class of elements

As pointed out in the introduction, this contribution can be linked to a discretisation setting presented in [12], where the considered space reads

$$
\begin{array}{r}
\mathcal{V}_{2, k}^{\text {face }}(K)=\left\{v \in H(\operatorname{div}, K) \cap H(\operatorname{curl}, K) \quad \text { s.t. }\left.\quad v \cdot n\right|_{f} \in \mathbb{P}_{k}(f) \forall f \in \partial K,\right. \\
\left.\operatorname{grad}(\operatorname{div}(v)) \in \nabla \mathbb{P}_{k-1}(K), \quad \text { and } \quad \operatorname{curl} v \in \mathbb{P}_{k-1}(K)\right\} .
\end{array}
$$

Restricting the setting on the boundary to polynomial functions, that is introducing

$$
\begin{gathered}
\tilde{\mathcal{V}}_{2, k}(K)=\left\{v \in H(\operatorname{div}, K) \cap H(\operatorname{curl}, K) \quad \text { s.t. }\left.\quad v \cdot n\right|_{f} \in \mathbb{P}_{k}(f) \quad \text { and }\left.\quad v\right|_{f} \in \underset{i=1}{X} \mathbb{Q}_{\zeta_{i}(k+1, k, \ldots, k)}(f) \quad \forall f \in \partial K,\right. \\
\left.\operatorname{grad}(\operatorname{div}(v)) \in \nabla \mathbb{P}_{k-1}(K), \quad \text { and } \quad \operatorname{curl} v \in \mathbb{P}_{k-1}(K)\right\} .
\end{gathered}
$$

It can in particular be shown that

$$
v \in \tilde{\mathcal{V}}_{2, k} \quad \Rightarrow \quad v \in \mathbb{H}_{k}(K),
$$

for the space $\mathbb{H}_{k}(K)$ is constructed from the coefficients $\left(l_{1}, l_{2}\right)=(0, k)$ and $\left(m_{1}, m_{2}\right)=(k,-1)$ as

$$
\begin{aligned}
\mathbb{H}_{k}(K)= & \left\{u \in H^{1}(K),\left.u\right|_{\partial K} \in \mathcal{H}_{0}(\partial K), \Delta u \in \mathbb{Q}_{k}(K)\right\}^{d} \\
& +x\left\{u \in H^{1}(K),\left.u\right|_{\partial K} \in \mathcal{H}_{k}(\partial K), \Delta u \in \mathbb{Q}_{[-1]}(K)\right\} .
\end{aligned}
$$

Indeed, any element $v \in \tilde{\mathcal{V}}_{2, k}(K)$ belongs to $H^{1}(K)$, and can be written as $v=v_{1}+v_{2}$, where $v_{1}$ lives in $A_{k}$ and $v_{2}$ lives in $x B_{k}$ (see the Appendix for a sketch of the proof). 
TABLE 3. Degrees of freedom of the element Ia defined within the reduced setting.

\begin{tabular}{cccc}
\hline \hline Core normal Moments & Misc moment & Core internal moment & Misc internal moment \\
\hline $\int_{f_{j}} x^{i} q \cdot n \mathrm{~d} \gamma$, & $\int_{f_{j}} q \cdot n \mathrm{~d} \gamma$ & $\int_{K}\left(\begin{array}{c}x^{l} y^{m} \\
0\end{array}\right) \cdot q \mathrm{~d} x \mathrm{~d} y$ and $\int_{K}\left(\begin{array}{c}0 \\
x^{m} y^{l}\end{array}\right) \cdot q \mathrm{~d} x \mathrm{~d} y$, & $\int_{K}\left(\begin{array}{c}x^{k} y^{k-1} \\
x^{k-1} y^{k}\end{array}\right) \cdot q \mathrm{~d} x \mathrm{~d} y$ \\
$\forall j \in \llbracket 1, \mathfrak{n} \rrbracket, i \in \llbracket 1, k \rrbracket$ & $j \in \llbracket 1, \mathfrak{n} \rrbracket$ & $\begin{array}{c}\forall l \in \llbracket 0, k \rrbracket, m \in \llbracket 0, k-1 \rrbracket \\
\text { s.t. }(l, m) \neq(k, k-1)\end{array}$ \\
\hline
\end{tabular}

The degrees of freedom selected in the framework of [12] are however different from what we do here. We allow more freedom in the inner characterisation while preserving the desired properties on the boundary. Note also that contrarily to the more general setting presented in this section, the lowest order elements of [12] cannot be natively defined. As the normal component on the boundary belongs at least to $\mathbb{P}_{1}(K)$ for any admissible space, the setting of [12] has to be slightly modified (see e.g. [4]).

\subsubsection{An example of a reduced setting}

As quickly addressed in the Section 3.2 and as it will be shown in the numerical results, a classical construction of the space $\mathbb{H}_{k}(K)$ implies the degeneration of some normal functions into internal ones. This is a consequence of the coordinate-wise freedom provided on the boundary from the definition of the set $A_{k}$. Therefore, to allow a parallel with the Raviart-Thomas elements from the lowest order on and to fulfil the optional Condition 3.4, one can consider replacing the boundary conditions $\left.u\right|_{\partial K} \in \mathcal{H}_{l_{1}}(\partial K)$ in $A_{k}$ to obtain the reduced space

$$
\begin{aligned}
\mathbb{H}_{k}(K)= & \left\{u \in H^{1}(K),\left.u\right|_{\partial K} \equiv 1, \Delta u \in \mathbb{Q}_{m_{1}}(K)\right\}^{d} \\
& \oplus x\left\{u \in H^{1}(K),\left.u\right|_{\partial K} \in \mathcal{H}_{l_{2}}(\partial K), \Delta u \in \mathbb{Q}_{\left[m_{2}\right]}(K)\right\} .
\end{aligned}
$$

There, the coordinate-wise freedom on the boundary is reduced and the normal degrees of freedom can be set as in the classical Raviart-Thomas setting. Furthermore, contrarily to the general case, any definition of $l_{2}, m_{1}$ and $m_{2}$ leads to an $H($ div)-conformity ready space.

\section{Example of a reduced two dimensional element}

To emphasise the parallel with the Raviart-Thomas setting on the boundary, we reduce the previous example and derive the corresponding reduced discretisation framework.

Definition 3.20 (Reduced space).

$$
\begin{aligned}
\mathbb{H}_{k}(K)= & \left\{u \in H^{1}(K),\left.u\right|_{\partial K} \equiv 1, \Delta u \in \mathbb{Q}_{k-1}(K)\right\}^{2} \\
& \oplus\left(\begin{array}{l}
x_{1} \\
x_{2}
\end{array}\right)\left\{u \in H^{1}(K),\left.u\right|_{\partial K} \in \mathcal{H}_{k}(\partial K), \Delta u \in \mathbb{Q}_{[k-1]}(K)\right\} .
\end{aligned}
$$

Its dimension then naturally reads:

$$
\operatorname{dim} \mathbb{H}_{k}(K)=\mathfrak{n}(k+1)+2 k(k-1)-\mathbb{1}_{k>0} .
$$

Therefore, exactly $k+1$ normal functions per edge can be designed, fitting the framework of Raviart-Thomas. As this matches the dimension of $\mathbb{Q}_{k}(f)$, all the freedom is required to entirely determine the global normal component. Thus, as a straightforward reduction of the general case, the $H(\operatorname{div}, K)$-conformal element presented in the Section 3.4 simplify to the following degrees of freedom (Table 3 ).

Note that here too, for defining the projections (3.18) one can work with any basis of $\mathcal{P}$ instead of the presented canonical basis. 

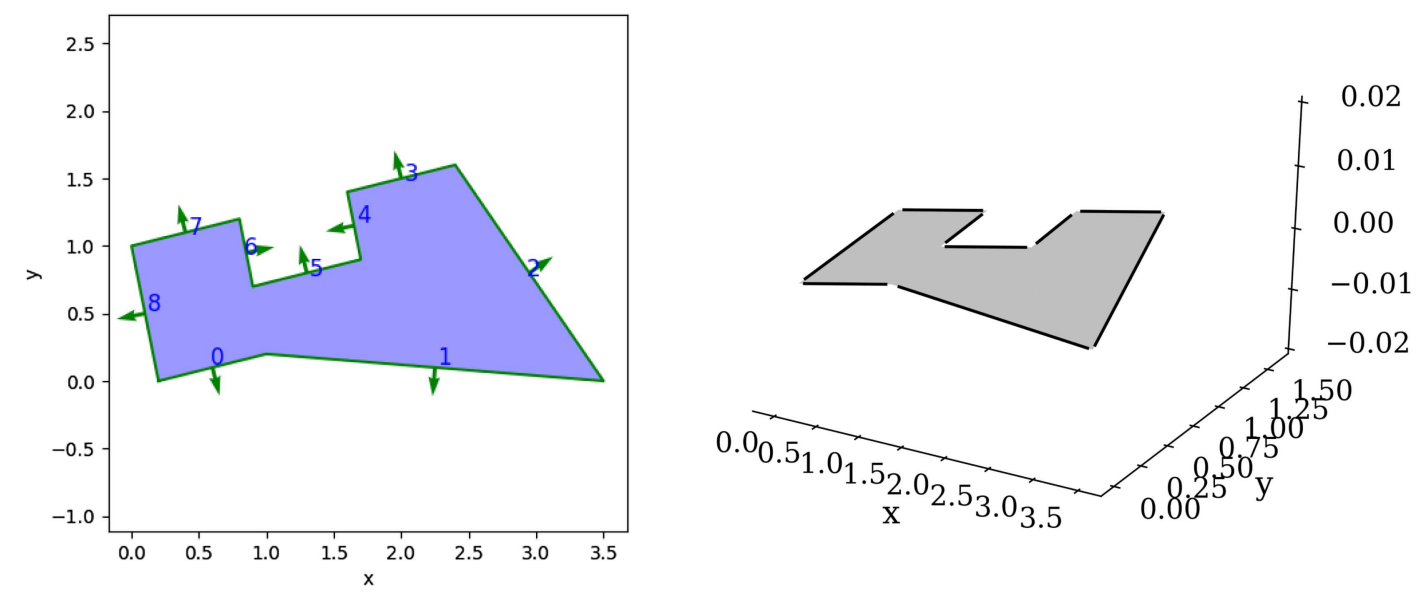

FIGURE 1. Left: considered polygon. Right: normal component of a representative internal basis functions plotted on every edge.

\section{Numerical Results}

We explore the properties of the main element Ia and its variant Ib presented in the previous section by investigating their basis functions, for both the general framework and the reduced one. We particularly focus on the normal component $\left.\varphi \cdot n\right|_{\partial K}$ of representative basis functions $\varphi$ on the boundary of the element $K$. Those basis functions have been constructed by tuning a natural basis of the space $\mathbb{H}_{k}(K)$ towards the selected sets of degrees of freedom through a transfer matrix.

As an example, we consider the non-convex nine-edges polygon presented in the Figure 1 on which the elements are built. In all the results, the polynomial projectors used in the definition of the degrees of freedom were chosen as Hermite polynomials: experimentally we have observed that this improves the conditioning of the linear system.

\subsection{General setting}

We start by considering the spaces and elements described in the Section 3.4.1. First of all, we have investigated the behaviour of the internal basis functions. The normal component of them is shown in the right of Figure 1. As wished, the basis functions corresponding to internal degrees of freedom vanish on the boundary. This can been seen on the right figure where the function is plotted in the plane $z=0$.

In order to study the behaviour of the normal basis functions on the boundaries, we have considered the case $k=2$ where we expect five basis functions to have a quadratic normal component. We have plotted in the left most side of the Figure 2 the normal component of one of the normal basis functions associated to the element Ib. One can observe that its support is contained on one single edge.

We then have plotted all the basis functions associated to the edge number 5 on the Figure 2, for both the configurations Ib (middle) and Ia (right). One can first notice that their normal components, plotted in the middle graph of the Figure 2, are polynomial of degree $k \leq 2$, that together generate the space $\mathbb{P}_{2}(\mathbb{R})$. Observing further, it appears that the normal component of two basis are vanishing, that is $\varphi \cdot n=\varphi_{1} \cdot n_{5, x}+\varphi_{2} \cdot n_{5, y}=0$. This generates this straight line equal to zero in the graph. Indeed, those two basis functions characterise the coordinate-wise freedom $\varphi_{1} \cdot n_{5, x} \neq 0$ and $\varphi_{2} \cdot n_{5, y} \neq 0$. This additional freedom is not reflected through the global term $\varphi \cdot n$ as addressed in the Example 3.11 and in the Remark 3.13. This comes from the fact that only three basis functions are required to generate $\mathbb{P}_{2}(\mathbb{R})$, where the global component $\varphi \cdot n$ lives. The two components 

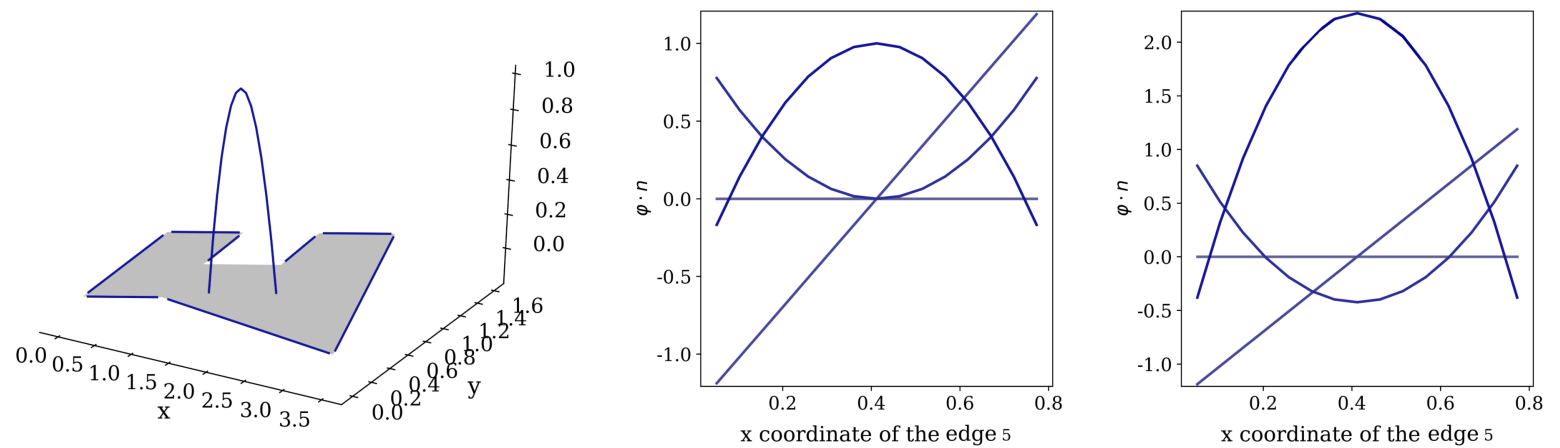

FIGURE 2. Left: normal component of one representative of the normal basis functions for the element $\mathrm{Ib}$ and $k=2$ along the edges. Middle: normal component of all the functions generated from the edge number 5, plotted on the edge number 5. Right: as middle, for the element Ia.
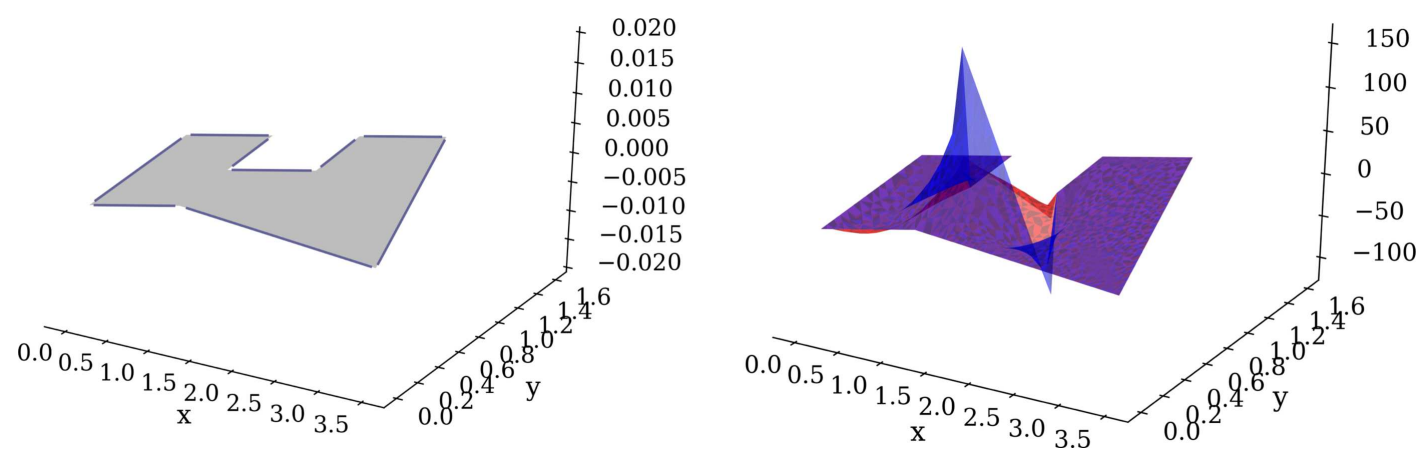

FiguRE 3. Degeneration of a degenerating normal basis functions' representative in the case $k=2$, for the element Ib. Left: normal component on all the boundaries. Right: internal behaviour of the basis functions.

$\varphi_{1} n_{5, x}$ and $\varphi_{2} n_{5, y}$ of the vector $\varphi \circ n$ are compensating themselves. Those functions are nevertheless regular within the polygon $K$ and not identically vanishing on $K$ (see Fig. 3, where the left graph represents the value of the normal component $\varphi \cdot n$ on the boundary and where the right graph represents the components $\varphi_{1}$ and $\varphi_{2}$ on the element $K)$. They can therefore be reclassified as internal basis functions. Note that this can be suppressed when using the reduced setting, as one can observe below in the Section 4.2.

Finaly, one can consider the scaling of the basis functions by plotting the normal basis functions corresponding to the configurations Ia and Ib in the lowest order case, i.e. for $k=0$ (see Fig. 4). There, only the configuration Ib using a point value scales to one. The fully moment-based configuration Ia scales to another constant that depends on the edge's length and orientation with respect to the axes. This example emphasises that the configuration Ib leads to basis functions which share similar properties like the Raviart-Thomas elements.

\subsection{Reduced setting}

As a last example, we derive some results obtained for the reduced element Ib, offering a complete parallel with the Raviart-Thomas elements on the boundary by suppressing the further coordinate-wise liberty provided by the general setting. The internal basis functions being unchanged from the general setting, they are not represented. 

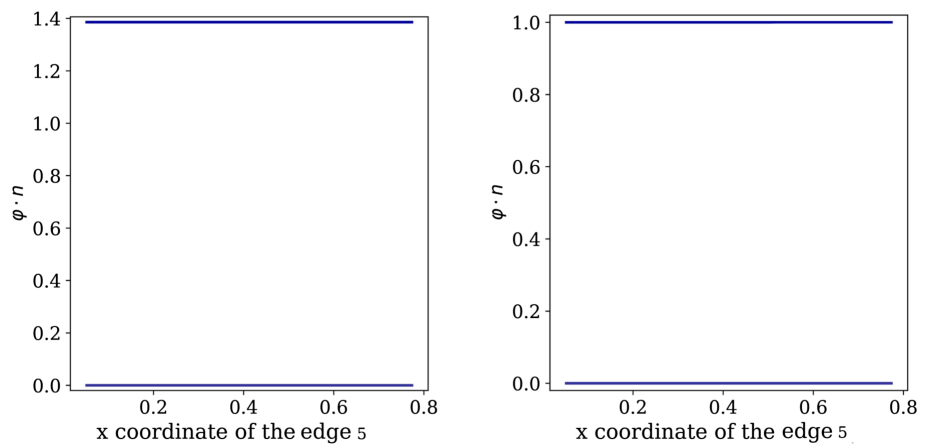

FiguRE 4. Scaling of the non-vanishing basis functions generated from the edge number 5 when $k=0$. From left to right: Ia and Ib.
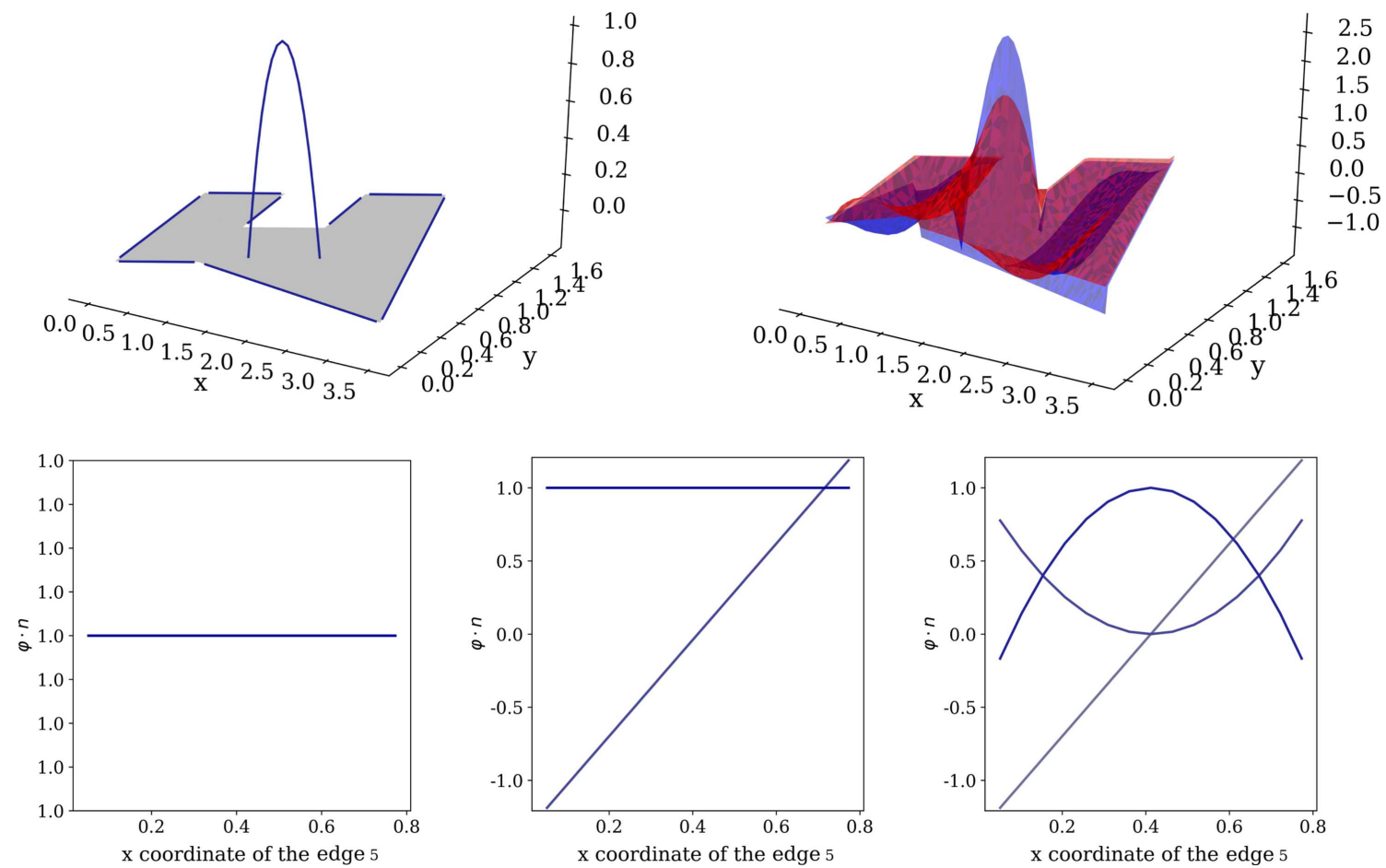

FiguRE 5. Top left: regularity of the components of one representative of the basis functions for the reduced element Ib and $k=2$ within the element. Top right: its normal component along the boundary. Bottom: normal component of all the functions generated from the edge number 5 , plot on the edge number 5 . From left to right: $k=0, k=1, k=2$.

Indeed, one can observe on the bottom of the Figure 5 that there is no more degenerating normal basis functions. Therefore, all normal basis functions are acting globally to characterise the polynomial behaviour of functions of the reduced $\mathbb{H}_{k}(K)$ space on the boundary. Furthermore, one can observe that the scaling of the basis functions corresponding to lowest order element, as well as the amplitude of the basis functions describing the higher order ones, make the discretisation framework reliable. 
Remark 4.1. The shape of the normal component of the basis functions is driven by the definition of the projectors $p$ in the normal degrees of freedom. Changing the basis of the projectors then allows to enforce wished shape of the basis functions of $\mathbb{H}_{k}(K)$ while keeping the regularity and order of the discretisation. Shifting them by modulating the offset directly from the definition of the degrees of freedom to enforce their positivity is equally possible.

\section{Conclusion}

Motivated by defining a flux reconstruction scheme on general polytopes [1], we have developed a new $H$ (div)conformal discretisation framework that can be set up on any polytope, not necessarily convex. It merges the flexibility of the Virtual Element setting with the properties of the Raviart-Thomas elements on the boundaries.

The introduced finite dimensional spaces are vectorial and allow a lot of flexibility in the definition of the degrees of freedom. In particular, the choices of discretisation quality and degrees of freedom on the boundary are independent from the ones made within the element.

The discretised quantities benefit from an extensive coordinate-wise freedom. Therefore, upon the choice made while selecting the degrees of freedom, some dual normal basis functions may be reclassified into internal ones. Thus, to allow a complete parallel with the Raviart-Thomas setting on the boundary from the lowest order on, one may construct straightforwardly a reduced space, along with reduced elements.

Last, we detailed a particular example of a discretisation framework through a series of spaces and the definition of a particular element. It could be observed that in both general and reduced frameworks, the type of degrees of freedom (point-wise values or moments) impacts the scaling of the dual basis functions. This can typically be observed in the lowest order case of the given examples, where only the dual basis functions of the element Ib scale to one.

An important topic for further research is the exploration of projectors from the introduced spaces onto polynomial ones, in a way similar to those already constructed in [12] and used in the Virtual Elements Methods. Especially, a suitable extension of those projectors to the spaces introduced here may be inferred from a close investigation of those projectors. Once the projectors have been defined, we can apply those discretisation spaces in a more practical context, as by example employing the introduced spaces in a finite element framework.

To conclude, let us point out again that the results presented here are already useful from a theoretical point of view. Indeed, they first guarantee that the considerations about FR schemes on general polytopes hold, and guarantee that the conjecture about the correction functions made in [1] is correct. Secondly, it opens the door to a more general framework in context of $\mathrm{FE}$, direction that will be further investigated in the future.

\section{Appendix A. A note on further possible COnfigurations}

As example, we only detail in this paper two declinations of one possible configuration of degrees of freedom. There exists many more possibilities, and their choice impact the properties of the elements. In particular, it is possible to focus on a coordinate-wise boundary characterisation of the quantities living in a general space $\mathbb{H}_{k}(K)$, rather than the global focus presented here. In the general setting, this choice yields a degeneracy of only one normal basis function, thus moving a bit away from the Raviart-Thomas spirit. For interested readers, a presentation of this possibility for both the general and the reduced space is available in [2], along with further investigations on various configurations.

Note also that different choices also have a very strong impact on the conditionning number of the linear systems to solve. The solution we have shown in this paper is the one that offers the best compromise. It is also the one that is the closest from the classical RT framework.

\section{Appendix B. Proofs}

Proof of Proposition 3.7. We start by deriving the first statement. By construction, any $q \in \mathbb{H}_{k}(K)$ can be decomposed into $q=q_{0}+x q_{1}$ for some $q_{0} \in\left(A_{k}\right)^{d}$ and $q_{1} \in B_{k}$. Therefore, on the boundary of $K$ one 
has $\left.q \cdot n\right|_{\partial K}=\left.q_{0} \cdot n\right|_{\partial K}+\left.\left(x q_{1} \cdot n\right)\right|_{\partial K}$. As the functions $q_{1}$ is scalar, this quantity can also read $\left.q \cdot n\right|_{\partial K}=$ $\left.q_{0} \cdot n\right|_{\partial K}+\left.q_{1}(x \cdot n)\right|_{\partial K}$ by linearity and commutativity of the dot product.

Since for every face $f$ of $K$ the term $\left.x \cdot n\right|_{f}$ is constant, it reduces to $\left.q \cdot n\right|_{f}=\left.q_{0} \cdot n\right|_{f}+\left.c_{f} q_{1}\right|_{f}$ on each face $f$ for a constant $c_{f} \in \mathbb{R}$ depending only on the face layout and position with respect to the axes and origin. Therefore, since $\left.q_{0}\right|_{f} \in\left(\mathbb{Q}_{l_{1}}(f)\right)^{d}$ and $\left.q_{1}\right|_{f} \in \mathbb{Q}_{l_{2}}(f),\left.q \cdot n\right|_{f} \in \mathbb{Q}_{\max \left\{l_{1}, l_{2}\right\}}(f)$. And since it is valid for any face $f \in \partial K$, we finally get that $\left.q \cdot n\right|_{\partial K} \in \mathbb{H}_{\max \left\{l_{1}, l_{2}\right\}}(\partial K)$

Let us now derive the divergence property within the cell. Any $u \in \mathbb{H}_{k}(K)$ can be written under the form $u=\tilde{q}+x q$ for some functions $q \in H^{1}(K)$ and $\tilde{q}=\left(\tilde{q_{1}}, \ldots, \tilde{q_{d}}\right)^{T} \in\left(H^{1}(K)\right)^{d}$ such that

$$
\left\{\begin{array} { r } 
{ \Delta q \in \mathbb { Q } _ { [ m _ { 2 } ] } ( K ) } \\
{ q | _ { \partial K } \in \mathbb { Q } _ { l _ { 2 } } ( \partial K ) }
\end{array} \quad \text { and } \quad \left\{\begin{array}{r}
\Delta \tilde{q}_{i} \in \mathbb{Q}_{m_{1}}(K) \\
\left.\tilde{q}_{i}\right|_{\partial K} \in \mathbb{Q}_{l_{1}}(\partial K),
\end{array} \quad \forall i \in \llbracket 1, d \rrbracket\right.\right.
$$

We have

$$
\begin{aligned}
\operatorname{div}(u) & =\sum_{i=1}^{d} \partial_{x_{i}}\left(x_{i} q\right)+\sum_{i=1}^{d} \partial_{x_{i}} \tilde{q}_{i}=\sum_{i=1}^{d}\left(q+x_{i} \partial_{x_{i}} q\right)+\sum_{i=1}^{d} \partial_{x_{i}} \tilde{q}_{i} \\
& =\underbrace{d q}_{\in L^{2}(K)}+\sum_{i=1}^{d}(x_{i} \underbrace{\partial_{x_{i}} q}_{\in L^{2}(K)})+\sum_{i=1}^{d} \underbrace{\partial_{x_{i}} \tilde{q}_{i}}_{\in L^{2}(K)} .
\end{aligned}
$$

Since by (B.1) we have $\nabla \cdot q \in L^{2}(K)$, it comes that for any $i \in \llbracket 1, d \rrbracket ; x_{i} \partial_{x_{i}} q \in L_{\text {loc }}^{2}(K)$. As $K$ is compact and bounded, we have $L_{\mathrm{loc}}^{2}(K)=L^{2}(K)$ and $\operatorname{div} q \in L^{2}(K)$. As a by-product, note that we can derive $\nabla \cdot(x \nabla q)=\nabla \cdot q+x \Delta q$, where $\Delta q \in \mathbb{Q}_{\max }\left[m_{1}, m_{2}+1\right]$ and $x \Delta q \in \mathcal{C}^{\infty}(K)$.

Lemma B.1. The configurations Ia and Ib are sets of degrees of freedom leading to unisolvent elements when endowed in $\mathbb{H}_{k}(K)$.

Proof of the Lemma B.1. We refer to the functions $p_{k}$ by the term "kernel", while using the term "integrand" to represent the term $q \cdot p_{k}$. Immediate transfer of this designation apply to the normal moment based degrees of freedom.

We first sketch the proof. To begin with, let us point out that the key lies in the Assumption 3.12 ensuring the linear independence of the set of point-wise values and moment's integrands. The linearity of the integral operators transfers then this independence to the moments themselves, characterising any function of $\mathbb{H}_{k}(K)$ independently on the boundary and within the cell. We proceed in three steps.

(1) First, we show that the internal characterisation of the function does not impact the normal one, allowing the determination to be done distinctively within the element and on the boundary.

(2) Then, we show that selecting the appropriate number of degrees of freedom in any of the sets Ia or Ib ensures a unique characterisation on the boundary. We use the fact that the kernels are scalar polynomials while the functions of $\mathbb{H}_{k}(K)$ are vector polynomials.

(3) Lastly, we consider the interior of the element where the characterisation is done through projections over linearly independent sets. Those projections of functions in $\mathbb{H}_{k}(K)$ are indeed neither identically null nor identically identical (i.e. they differ at least on a subset of non-zero measure).

Let us detail this determination process more in details.

Step 1. Let us first recall that the space $\mathbb{H}_{k}(K)$ is built from blocks of independent functions. In particular, the boundary behaviour of functions living in $\mathbb{H}_{k}(K)$ is independent of their behaviour within the inner cell. Therefore, by the structure of $\mathbb{H}_{k}(K)$ and making use of the superposition theorem, any function $q \in \mathbb{H}_{k}(K)$ reads

$$
q=f \mathbb{1}_{\partial K}+g \mathbb{1}_{K}
$$

for two functions $f$ and $g$ belonging to $\mathbb{H}_{k}(K)$. As a consequence, characterising a function $q \in \mathbb{H}_{k}(K)$ comes down to characterising the independent functions $f$ and $g$ on the distinct supports $\partial K$ and $K$, 
respectively. Note also that necessarily, $\left.f\right|_{f_{j}} \in X_{i=1}^{d} \mathbb{Q}_{\max \left\{l_{1}, l_{2}+1\right\}}\left(\mathbb{R}^{d-1}\right)$ for any face $f_{j} \in \partial K$. We show that any admissible extraction (in the sense of the Admissibility conditions 3.12) from either of the two sets of degrees of freedom (Ia, internal), (Ib, internal) fully characterises the functions $f$ and $g$, independently. In all the following, the notation Ia or Ib refers to the corresponding set of normal degrees of freedom while "internal" refers to the set (3.14) and is identical to any of the two configurations under consideration.

We first show that any above defined set of degrees of freedom preserve the independence of the boundary and inner characterisations. To this aim, we combine the relation (B.2) with the all possible definitions of the degrees of freedom. It comes that all global normal moments lead to an expression of the form

$$
\sigma(q)=\int_{f_{j}} q \cdot n p_{k}=\int_{f_{j}}\left(f \mathbb{1}_{f_{j}}+g \mathbb{1}_{K}\right) \cdot n p_{k}=\int_{f_{j}} f \cdot n p_{k}
$$

for some polynomial function $p_{k}$ living on $\partial K$. On the other side, as $x_{j m} \in f_{j}$, the global degrees of freedom that are built from point-wise values read

$$
\sigma(q)=q\left(x_{j m}\right) \cdot n=f\left(x_{j m}\right) \cdot n \mathbb{1}_{f_{j}}\left(x_{j m}\right)+g\left(x_{j m}\right) \cdot n \mathbb{1}_{K}\left(x_{j m}\right)=f\left(x_{j m}\right) \cdot n .
$$

Similar relations for coordinate - wise degrees of freedom can be derived, that is;

$$
\text { and } \begin{aligned}
\sigma(q) & =\int_{f_{j}} q_{x_{i}} n_{x_{i}} p_{k}=\int_{f_{j}}\left(f_{x_{i}} \mathbb{1}_{f_{j}}+g_{x_{i}} \mathbb{1}_{K}\right) n_{x_{i}} p_{k}=\int_{f_{j}} f_{x_{i}} n_{x_{i}} p_{k} \\
& =q_{x_{i}}\left(x_{j m}\right) n_{x_{i}}=f_{x_{i}}\left(x_{j m}\right) n_{x_{i}} \mathbb{1}_{f_{j}}\left(x_{j m}\right)+g_{x_{i}}\left(x_{j m}\right) n_{x_{i}} \mathbb{1}_{K}\left(x_{j m}\right) \\
& =f_{x_{i}}\left(x_{j m}\right) n_{x_{i}},
\end{aligned}
$$

where here the terms $f_{x_{i}}$ simply represent the $i$ th component of the function $f$. Therefore, in any of the configurations Ia and Ib no contribution of the function $g$ representing the inner part of the cell is involved in the normal degrees of freedom. The mirror case is obtained with the internal moments, leading via (B.2) to

$$
\sigma(q)=\int_{K} q \cdot p_{k}=\int_{K}\left(f \mathbb{1}_{\partial K}+g \mathbb{1}_{K}\right) \cdot p_{k}=\int_{K} g \cdot p_{k},
$$

where $p_{k}$ stands for any Poisson's function living in $\mathbb{H}_{k}(K)$ or any polynomial function defining the second member of a Poisson's problem involved in the definition of $\mathbb{H}_{k}(K)$. There, the function $f$ representing the boundary part of the function $q$ is not involved, that for any definition of the space $\mathcal{P}_{k}$ generating the internal moments. Thus, by linearity we can decompose the degrees of freedom $\left\{q \mapsto \sigma_{i}(q)\right\}_{i}$ in the following matrix.

$$
\underset{\text { Nolues }}{\substack{\text { Internal Dofs } \\
\text { values }}}\left\{\left(\begin{array}{c}
\sigma_{1} \\
\vdots \\
\sigma_{N_{N}} \\
\sigma_{N_{N}+1} \\
\vdots \\
\sigma_{N_{I}}
\end{array}\right)=\left(\begin{array}{cccc}
\text { Normal } & \text { moments } & 0 & \\
\text { applied to } & f & & \\
& & \text { Internal } & \text { moments } \\
0 & & \text { applied to } & g
\end{array}\right)\left(\begin{array}{c}
f \\
g
\end{array}\right)\right.
$$

Clearly, there is no interconnection between the function's characterisation on the boundaries and the one performed within the element. Thus, showing the Proposition 3.19 reduces to show independently that Ia $=$ 0 or $\mathrm{Ib}=0$ implies $\left.f\right|_{\partial K}=0$ and $\int_{K} g \cdot p_{k} x=0$, for all $p_{k} \in \mathcal{P}_{k}$ implies $\left.g\right|_{K}=0$.

Step 2. Let us now consider the boundary characterisation. There, by definition of the spaces $\mathcal{H}_{l_{1}}$ and $\mathcal{H}_{l_{2}}$, the function $\left.f\right|_{\partial K}$ is discontinuous at the polytope's vertices and can be decomposed into $n$ vectorial polynomial 
functions $\left\{f_{j}\right\}_{j}$ with distinct supports, each of them matching one particular face of the polytope. Thus, we can write

$$
\left.f\right|_{\partial K}=\sum_{j=1}^{n} r_{j} \mathbb{1}_{f_{j}}
$$

with $r_{j} \in X_{i=1}^{d} \mathbb{Q}_{\max \left\{l_{1}, l_{2}+1\right\}}\left(f_{j}\right)$ and $f_{j}$ any face belonging to $\partial K$. With a similar argument than in the previous point, the characterisation of $\left.f\right|_{\partial K}$ can therefore be done edge-wise, and the determination matrix becomes block - diagonal. We discuss here the characterisation on one particular edge $f_{j}$ by showing the invertibility of the corresponding matrix block. The arguments naturally transpose to the other ones.

In this perspective, let us show that for any $r_{j} \in X_{i=1}^{d} \mathbb{Q}_{\max \left\{l_{1}, l_{2}+1\right\}}\left(f_{j}\right)$, it holds $\left\{\left.(I a)\right|_{f_{j}}=0\right.$ or $\left.(I b)\right|_{f_{j}}=$ $0\} \Rightarrow r_{j}=0$, where $\left.(\cdot)\right|_{f_{j}}$ represents the subset of the degrees of freedom $(\cdot)$ whose support (or evaluation point for point-values) matches (or lies on) $f_{j}$.

First of all, we recall that on the face $f_{j}$ the function $r_{j}$ is a multi-valued polynomial of the form

$$
\left.r_{j}\right|_{f_{j}}=\left(\begin{array}{c}
a_{0,1} \\
\vdots \\
a_{0, d}
\end{array}\right)+\sum_{i=\operatorname{dim}\left(\mathcal{H}_{0}\right)}^{\operatorname{dim}\left(\mathcal{H}_{l_{1}} \cap \mathcal{H}_{l_{2}}\right)}\left(\begin{array}{c}
b_{\xi_{1}(i)}+a_{i, 1} \\
\vdots \\
b_{\xi_{d}(i)}+a_{i, d}
\end{array}\right) m_{\alpha_{i}(x)}+\sum_{i=\operatorname{dim}\left(\mathcal{H}_{l_{1}} \cap \mathcal{H}_{l_{2}}\right)}^{\operatorname{dim}\left(\mathcal{H}_{l_{2}}\right)}\left(\begin{array}{c}
x_{1} b_{i} \\
\vdots \\
x_{d} b_{i}
\end{array}\right) m_{\alpha_{i}(x)},
$$

where $m_{\alpha_{i}}$ represents a monomial of $\mathbb{Q}_{\max \left\{l_{1}, l_{2}\right\}}$ of multi-index degree $\alpha_{i}$ such that the set $\left\{m_{\alpha_{i}}\right\}_{i=\operatorname{dim}\left(\mathcal{H}_{l}\right)}^{\operatorname{dim}\left(\mathcal{H}_{m}\right)}$ forms a base of $\mathcal{H}_{m} \backslash \mathcal{H}_{l}$. Note that the coefficients $\left\{a_{i j}\right\}_{i, j}$ are defined coordinate-wise while the coefficients $\left\{b_{i}\right\}_{i}$ are identical for all the components. The function $r_{j}$ is therefore determined by

coefficients.

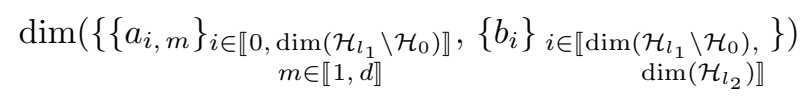

As in all configurations the function $r_{j}$ is determined only through its normal components, let us use the above expression to derive them more specifically. With the normal $n_{j}=\left(n_{j x_{1}, \ldots, n_{j x_{d}}}\right)$ to the face $f_{j}$, it comes

$$
\left.r_{j} \cdot n_{j}\right|_{f_{j}}=\sum_{m=1}^{d} a_{0, m} n_{j x_{m}}+\sum_{i=\operatorname{dim}\left(\mathcal{H}_{0}\right)}^{\operatorname{dim}\left(\mathcal{H}_{l_{1}} \cap \mathcal{H}_{l_{2}}\right)} \sum_{m=1}^{d}\left(b_{\xi_{m}(i)}+a_{i, m}\right) n_{j x_{m}} m_{\alpha_{i}}(x)+\sum_{i=\operatorname{dim}\left(\mathcal{H}_{l_{1}} \cap \mathcal{H}_{l_{2}}\right)}^{\operatorname{dim}\left(\mathcal{H}_{l_{2}}\right)} c_{j} b_{i} m_{\alpha_{i}}(x),
$$

where $c_{j}=x \cdot n_{j}$ is a constant term on the face $f_{j}$. Reordering the terms, we end up with the formulation

$$
\begin{aligned}
& \left.r_{j} \cdot n\right|_{f_{j}}=\sum_{m=1}^{d}\left(\left(\begin{array}{c}
\operatorname{dim}\left(\mathcal{H}_{l_{1}} \cap \mathcal{H}_{l_{2}}\right) \\
\left.\left.a_{0, m}+\sum_{i=\operatorname{dim}\left(\mathcal{H}_{0}\right)} a_{i, m} m_{\alpha_{i}(x)}\right) n_{j x_{m}}\right)
\end{array}\right.\right. \\
& +\left(\sum_{m=1}^{d} n_{j x_{m}}\right)^{\operatorname{dim}\left(\mathcal{H}_{l_{1}} \cap \mathcal{H}_{l_{2}}\right)} \sum_{i=1}^{b_{\xi_{m}}(i)} m_{\alpha_{i}}(x)+c_{j}\left(\sum_{i=\operatorname{dim}\left(\mathcal{H}_{l_{1}} \cap \mathcal{H}_{l_{2}}\right)}^{\operatorname{dim}\left(\mathcal{H}_{l_{2}}\right)} b_{i} m_{\alpha_{i}}(x)\right) .
\end{aligned}
$$

The structure of the retrieved form makes clearly emerge the coefficients that should be used depending on the coordinate-wise behaviour of the polygon.

In addition, as all the coefficients determining $r_{j}$ appear in this expression, using degrees of freedom defined only from the normal components of tested functions is admissible. Thus, the two configurations fitting this framework, we only have to make sure that the set of extracted degrees of freedom are uniquely characterising each of the involved coefficients. To this aim, we explicit all the possible degrees of freedom when applied to the function $r_{j}$. For the sake of clarity, we denote by $\left\{\sigma_{M_{i, l}}\right\}_{i l}$ the moments designed coordinate-wise, being of the form

$$
\sigma_{M_{i, l}}: q \mapsto q_{x_{i}}\left(x_{j l}\right) n_{j x_{i}} \quad \text { or } \quad \sigma_{M_{i, l}}: q \mapsto \int_{f_{j}} q_{x_{i}} n_{j x_{i}} p_{l}
$$


for some scalar polynomial $p_{l}$, and by $\left\{\sigma_{T_{l}}\right\}_{l}$ the ones acting globally, reading

$$
\sigma_{T_{l}}: q \mapsto q\left(x_{l}\right) \cdot n \quad \text { or } \quad \sigma_{T_{l}}: q \mapsto \int_{f_{j}} q \cdot n p_{l}
$$

for some scalar polynomial $p_{l}$. Further, for convenience we denote by $\left\{\sigma_{V_{l}}\right\}_{l}$ the global degrees of freedom that comes into play to determining the coordinate - wise coefficients, whose expressions are done in the same way as $\left\{\sigma_{T_{l}}\right\}_{l}$. We now express those degrees of freedom depending on the coefficients $\left\{b_{i, m}\right\}_{i m}$ and $\left\{a_{i}\right\}_{i}$. Using the linearity of the degrees of freedom, plugging the expression (B.3) in place of $q$ and setting the permutation operator directly on the multi - indices $\alpha_{i}$ instead of the coefficients $a_{i}$, we can rewrite the moments as follows.

$$
\begin{aligned}
\sigma_{M_{m, l}}:\left(\left\{a_{i, m}\right\},\left\{b_{i}\right\}\right) \longmapsto & b_{0, m} \int_{f_{j}} n_{j x_{m}} p_{l}+\sum_{i=\mathcal{H}_{0}}^{\operatorname{dim}\left(\mathcal{H}_{l_{1}} \cap \mathcal{H}_{l_{2}}\right)} a_{i, m} \int_{f_{j}} m_{\alpha_{i}}(x) n_{j x_{m}} p_{l} \\
& \operatorname{dim}\left(\mathcal{H}_{l_{1}} \cap \mathcal{H}_{l_{2}}\right) \\
& +\sum_{i=\mathcal{H}_{0}} b_{i} \int_{f_{j}} n_{j x_{m}} m_{\xi_{m}\left(\alpha_{i}\right)}(x) p_{l}+\sum_{i=\operatorname{dim}\left(\mathcal{H}_{l_{1}} \cap \mathcal{H}_{l_{2}}\right)}^{\operatorname{dim}\left(\mathcal{H}_{l_{2}}\right)} b_{f_{j}}\left(x_{m} n_{j x_{m}} m_{\alpha_{i}}(x)\right) p_{l} \\
\sigma_{T_{l}}:\left(\left\{a_{i, m}\right\},\left\{b_{i}\right\}\right) \longmapsto & \sum_{m=1}^{d} a_{0, m} \int_{f_{j}} n_{j x_{m}} p_{l}+\sum_{m=1}^{d \operatorname{dim}\left(\mathcal{H}_{l_{1}} \cap \mathcal{H}_{l_{2}}\right)} \sum_{i=\operatorname{dim}\left(\mathcal{H}_{0}\right)} a_{i, m} \int_{f_{j}} m_{\alpha_{i}}(x) n_{j x_{m}} p_{l} \\
& \operatorname{dim}\left(\mathcal{H}_{\left.l_{1} \cap \mathcal{H}_{l_{2}}\right)} d\right. \\
& +\sum_{i=\mathcal{H}_{0}} b_{i} \sum_{m=1}^{\operatorname{dim}\left(\mathcal{H}_{l_{2}}\right)}\left(\int_{f_{j}} n_{j x_{m}} m_{\xi_{m}\left(\alpha_{i}\right)}(x) p_{l}\right)_{i=\operatorname{dim}\left(\mathcal{H}_{l_{1}} \cap \mathcal{H}_{l_{2}}\right)} \int_{f_{j}}\left(c_{j} m_{\alpha_{i}}(x) p_{l}\right) .
\end{aligned}
$$

Thus, defining the component-wise parts of the global moments $\sigma_{T_{l}}$ by $\sigma_{T_{m, l}}(q)=\int_{f_{j}} n_{j x_{m}} q p_{l}$ such that $\sigma_{T_{l}}=\sum_{m=1}^{d} \sigma_{T_{m, l}}$, one can express any degrees of freedom of the two considered sets as

$$
\begin{aligned}
& \sigma_{M_{m, l}}: q \longmapsto a_{0, m} \sigma_{M_{m, l}}(1)+\sum_{i=\mathcal{H}_{0}}^{\operatorname{dim}\left(\mathcal{H}_{l_{1}} \cap \mathcal{H}_{l_{2}}\right)} a_{i, m} \sigma_{M_{m, l}}\left(m_{\alpha_{i}}\right) \\
& +\sum_{i=\mathcal{H}_{0}}^{\operatorname{dim}\left(\mathcal{H}_{l_{1}} \cap \mathcal{H}_{l_{2}}\right)} b_{i} \sigma_{M_{m, l}}\left(m_{\xi_{m}\left(\alpha_{i}\right)}\right)+\sum_{i=\operatorname{dim}\left(\mathcal{H}_{l_{1}} \cap \mathcal{H}_{l_{2}}\right)}^{\operatorname{dim}\left(\mathcal{H}_{l_{2}}\right)} b_{i} \sigma_{M_{m, l}}\left(x_{m} m_{\alpha_{i}}\right) \\
& \text { and } \quad \sigma_{T_{l}}: q \longmapsto \sum_{m=1}^{d} a_{0, m} \sigma_{T_{m, l}}(1)+\sum_{i=\mathcal{H}_{0}}^{\operatorname{dim}\left(\mathcal{H}_{l_{1}} \cap \mathcal{H}_{l_{2}}\right) d} \sum_{m=1}^{d} a_{i, m} \sigma_{T_{m, l}}\left(m_{\alpha_{i}}\right) \\
& +\sum_{i=\mathcal{H}_{0}}^{\operatorname{dim}\left(\mathcal{H}_{l_{1}} \cap \mathcal{H}_{l_{2}}\right)} b_{i} \sum_{m=1}^{d} \sigma_{T_{m, l}}\left(m_{\xi_{m}\left(\alpha_{i}\right)}\right)+\sum_{i=\operatorname{dim}\left(\mathcal{H}_{l_{1}} \cap \mathcal{H}_{l_{2}}\right)}^{\operatorname{dim}\left(\mathcal{H}_{l_{2}}\right)} a_{i} c_{j} \sigma_{T_{l}}\left(m_{\alpha_{i}}\right) .
\end{aligned}
$$

Note that in view of deriving the determination matrix, the last term can also be decomposed as follows.

$$
\sum_{i=\operatorname{dim}\left(\mathcal{H}_{l_{1}} \cap \mathcal{H}_{l_{2}}\right)}^{\operatorname{dim}\left(\mathcal{H}_{l_{2}}\right)} b_{i} c_{j} \sigma_{T_{l}}\left(m_{\alpha_{i}}\right)=\sum_{i=\operatorname{dim}\left(\mathcal{H}_{l_{1}} \cap \mathcal{H}_{l_{2}}\right)}^{\operatorname{dim}\left(\mathcal{H}_{l_{2}}\right)} b_{i=1}^{d} \sigma_{T_{l, m}}\left(x_{m} m_{\alpha_{i}}\right) .
$$

Similar relations for $\sigma_{V}$ can ve derived from the expression of $\sigma_{T}$. Thus, we can rewrite the degrees of freedom as a dot product and derive the characterisation matrix $\Sigma$

$$
\left(\sigma_{M_{1,1}}, \ldots, \sigma_{M_{d, l}}, \sigma_{V_{1}}, \ldots, \sigma_{V_{l}}, \sigma_{T_{1}}, \ldots, \sigma_{T_{l}},\right)^{T}=\Sigma\left(\left\{a_{i, m}\right\}_{i m},\left\{b_{i}\right\}_{i}\right)^{T}
$$

which shape is given in the Figure B.1. We now investigate its structure. 
First of all, as the number of extracted degrees of freedom from the two sets Ia and Ib matches the number of coefficients determining $r_{j}$, the matrix $\Sigma$ is a square matrix.

Let us focus on the top two-by-two left blocks, surrounded in blue. They correspond to the coefficients that should be determined coordinate-wise. Thus, by construction, there are $\operatorname{dim}\left(\left\{a_{i m}\right\}_{i m}\right)=d\left(l_{1}+1\right)^{d-1}$ columns. And by the definition of the configurations II and I, we have $\operatorname{dim}\left(\left\{\sigma_{M_{i, j}}\right\}_{i j}\right)=d+d\left(\left(l_{1}+1\right)^{d-1}-1\right)=$ $d\left(l_{1}+1\right)^{d-1}$. Therefore, this submatrix is a square matrix. Furthermore, each subblock corresponds to one member of the decomposition of $q$ tested through coordinate - wise degrees of freedom whose kernels are built on the same monomial. Therefore, as the degrees of freedom $\left\{\sigma_{M_{i, j}}\right\}$ consider one normal component only, the coefficients $\left\{a_{i m}\right\}_{i m}$ for $m \neq j$ are not involved, and the subblocks are diagonal. Thus, those submatrices are invertible and in particular their columns and rows are linearly independent.

On the other side of the matrix, the last bottom block surrounded in deep red matches the Raviart-Thomas moments tuning members of $\left.\mathbb{H}_{k}(K)\right|_{\partial K}$ living exclusively in $\left.x B_{k}\right|_{f_{j}}$. It is then a submatrix of the classical Raviart-Thomas' one, and is thus invertible. In particular, its rows and columns are linearly independent.

The extended bottom right submatrix highlighted in dashed red corresponds to the previously described high-order submatrix of the Raviart-Thomas's setting, enriched by the moments $\left\{\sigma_{V}\right\}$ tuning the behaviour of members of $\left.\mathbb{H}_{k}(K)\right|_{f_{j}}$ falling in the intersection $\mathcal{H}_{l_{1}} \cap \mathcal{H}_{l_{2}}\left(f_{j}\right)$.

This matrix is equivalent to the full Raviart-Thomas setting. Indeed, even if the moments $\left\{\sigma_{V}\right\}$ have to be slightly modified from the Raviart-Thomas setting in the configuration $I$, this modification leaves the projection order unchanged and the integrand still belongs to $\left.\mathbb{H}_{k}(K)\right|_{f_{j}}$. Therefore, the dashed line block is invertible and its columns and rows are linearly independent.

There is only left to show that there is no linear dependence between rows of different row blocks. As the degrees of freedom are linear forms, it is enough to show that the integrand of moments (or polynomials constructing the point - wise values) that involve the same monomial are linearly independent.

Indeed, being linear forms whose kernels are polynomials, the degrees of freedom can combine each other only if their integrand ( $q$ tested against the kernel) involve - up to constants - the same monomials. We then have to show that in both configurations, the rows involving terms whose projection onto the kernel can be expressed from a same monomial are linearly independent.

In the configurations Ia and Ib, this property comes automatically. Indeed, the only interaction between degrees of freedom having integrands sharing the same monomial order (and then possibly being based on the same monomial) is possible between (3.11a) and (3.11b) when $\left|p_{k}\right|=l_{1}+1$. Indeed, by definition of $\mathbb{H}_{k}(K)$, the polynomial $p_{k} \cdot n$ in (3.11a) is only of order $l_{1}$. However, no combination of (3.11b) can form the moments (3.11a). Indeed, for any real coefficients $c_{i}$ it holds

$$
\sum c_{i} q_{x_{i}} n_{x_{i}} x_{i}^{l_{1}+1} \not \equiv q \cdot n p_{k}
$$

for any monomial $p_{k}$ such that $\left|p_{k}\right|=l_{1}+1$. Note that in the left hand side, all the $c_{i}$ should be non-null to reconstruct the term $p \cdot n$. However, doing so no factorisation by a single monomial such that

$$
\sum_{i=1}^{d} c_{i} q_{x_{i}} n_{x_{i}} x_{i}^{l_{1}+1}=\left(\sum_{i=1}^{d} c_{i} q_{x_{i}} n_{x_{i}}\right) p_{k}
$$

is possible.

Thus, the designed moments are linearly independent, and no row combination can occur for any tested polynomial belonging to $\left.\mathbb{H}_{k}(K)\right|_{\partial K}$.

All in all, for both configurations all the rows are linearly independent. As by construction we have as many relations as unknowns, the matrix is invertible. Thus, we get a null kernel, meaningly

$$
I a=0 \text { or } I b=0 \text { implies }\left.f\right|_{\partial K}=0 .
$$

Step 3. Let us now consider the internal characterisation of functions living in $\mathbb{H}_{k}(K)$. From the first point of the proof, it is enough to study the characterisation of $g$ within the inner cell. By definition of $\mathbb{H}_{k}(K)$, any 


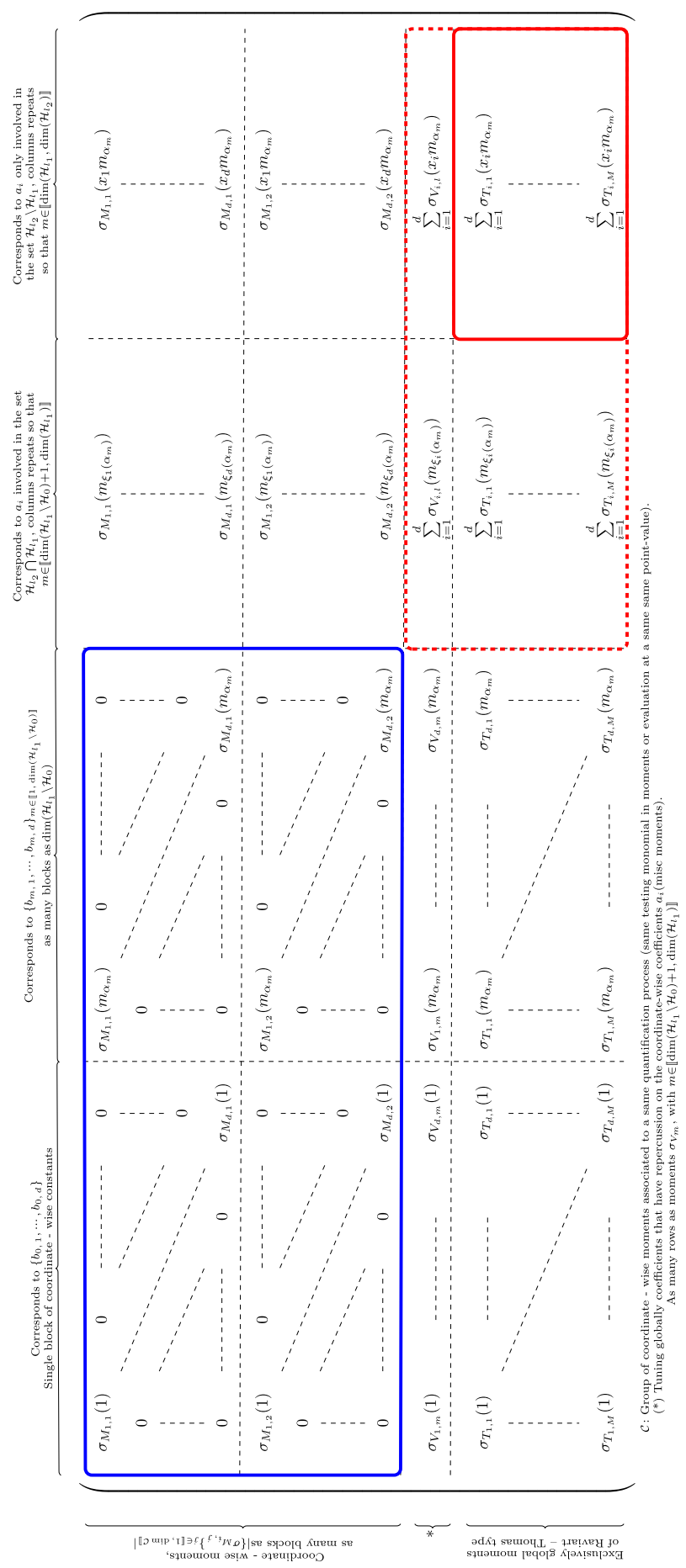

Figure B.1. Disposition of the degrees of freedom. 
function $\left.g \in \mathbb{H}_{k}(K)\right|_{K}$ can be decomposed over a set of Poisson's solutions as follows:

$$
g=\sum_{i=1}^{d} \sum_{i=1}^{\operatorname{dim} A_{k}} a_{i, j} e_{j} u_{i}+\sum_{i=1}^{\operatorname{dim} B_{k}} b_{j} x \tilde{u}_{i} .
$$

Here, the vector $e_{j}$ stands for $e_{j}=(0, \ldots, 1,0, \ldots, 0)^{T}$ where the 1 is in the $j$ th position. The functions $u_{i}$ and $\tilde{u}_{i}$ represents the Poisson's solutions of the problems

$$
\left\{\begin{array} { l } 
{ \Delta u _ { i } = p _ { i } , p _ { i } \in \mathbb { Q } _ { m _ { 1 } } ( K ) } \\
{ u _ { i } | _ { \partial K } = 0 }
\end{array} \text { and } \left\{\begin{array}{l}
\Delta \tilde{u}_{i}=\tilde{p}_{i}, \tilde{p}_{i} \in \mathbb{Q}_{\left[m_{2}\right]}(K) \\
\left.\tilde{u}_{i}\right|_{\partial K}=0
\end{array}\right.\right.
$$

where $\left\{p_{i}\right\}_{i}$ and $\left\{\tilde{p}_{i}\right\}_{i}$ form respectively a basis of $\mathbb{Q}_{m_{1}}(K)$ and $\mathbb{Q}_{\left[m_{2}\right]}(K)$. In any presented definition of the degrees of freedom, the internal characterisation is done through moment - based degrees of freedom of the form

$$
\sigma_{I_{k}}: q \mapsto \int_{K} q \cdot p_{k} \mathrm{~d} x
$$

where the kernels $p_{k} \in \mathcal{P}_{k}$ consist of linearly independent polynomials belonging to $\mathbb{Q}_{\max \left\{m_{1}, m_{2}+1\right\}}(K)$, or of the solution of their corresponding problems of the form (B.4). Therefore, we can derive a characterisation matrix in the same spirit as in the case of the normal characterisation.

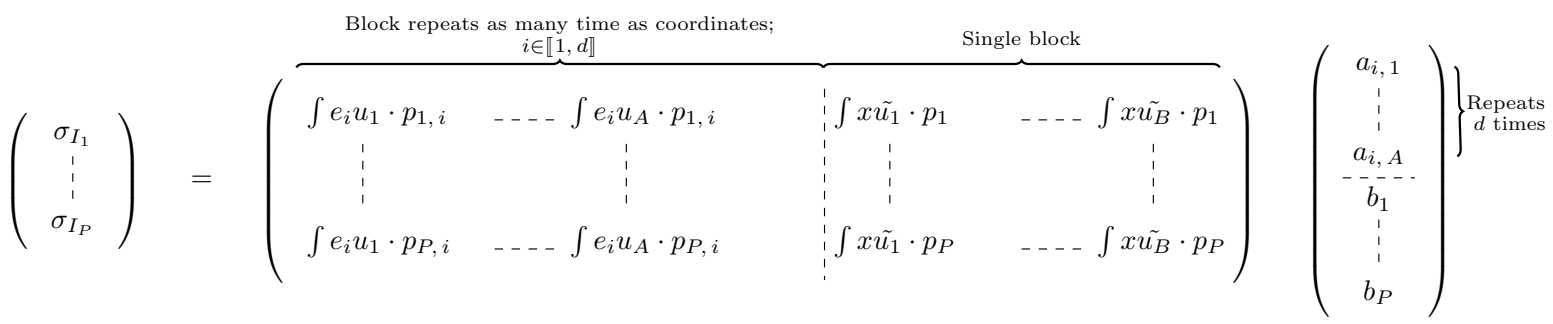

Let us consider the case where $\mathcal{P}_{k}$ forms a polynomial projection space. There, none of the $p_{k} \in \mathcal{P}_{k}$ is the zero function. In the same time, the functions $\left\{\left\{u_{i}\right\}_{i},\left\{x \tilde{u}_{i}\right\}_{i}\right\}$ are linearly independent, and being solutions to some Poisson's problem with non-zero second member, they are by construction not identically vanishing on $K$. Indeed, even when $m_{2}<m_{1}$ where second members of the problems (B.4) lives both in $\mathbb{Q}_{m_{1}}$ and $\mathbb{Q}_{\left[m_{2}\right]}$, it holds $\Delta\left(x \tilde{u}_{i}\right)=2 \nabla \cdot \tilde{u}_{i}+\Delta\left(\tilde{u}_{i}\right)$. Thus, it is impossible to combine linearly the function $x \tilde{u}_{i}$ with functions of the set $\left\{u_{i}\right\}_{i}$.

Furthermore, the degrees of the polynomials belonging to the space $\mathcal{P}_{k}$ are lower or equal than the highest degree of the second members of the Poisson's problem defining the space $\mathbb{H}_{k}(K)$. Thus, every projection of function of $\mathbb{H}_{k}(K)$ onto the space $\mathcal{P}_{k}$ is not null. And as the internal moments are linear forms, any linear combination of those moments at fixed $p_{k}$ could have its integrand factorised by the kernel $p_{k}$ for any $p_{k} \in \mathcal{P}_{k}$, transferring the linear independency of the set $\left\{\left\{u_{i}\right\}_{i},\left\{x \tilde{u}_{i}\right\}_{i}\right\}$ to the terms $\left\{\int u_{i} \cdot p_{k}\right\}_{i}$ for any fixed $p_{k} \in \mathcal{P}_{k}$.

Lastly, as the space $\mathcal{P}_{k}$ contains only linearly independent functions the previous argument can be repeated for each row of the matrix defined in (B.5). And as by construction the number of internal degrees of freedom matches the dimension of the space $\left.\mathbb{H}_{k}(K)\right|_{K}$, the linear independence of functions of $\mathcal{P}_{k}$ combined with the linear independence of the tested functions transfer automatically to the moments tested against a basis of $\left.\mathbb{H}_{k}(K)\right|_{K}$. Thus, the internal submatrix is invertible. The same reasoning can be applied when $\mathcal{P}_{k}$ is built from the Poisson's solutions themselves, as the projections of functions would decompose the functions directly.

Merging the above points together, we get that $I a=0$ or $I b=0$ implies $\left.f\right|_{\partial K}=0$ and $\int_{K} g \cdot p_{k} x=0$ for all $p_{k} \in \mathcal{P}_{k}$ implies $\left.g\right|_{K}=0$. From this, we get that for $q \in \mathbb{H}_{k}(K) I a=0$ or $I b=0$ and $\int_{K} q \cdot p_{k} x=0$ for all $p_{k} \in \mathcal{P}_{k}$ implies $q=0$. 
Proof of the Propositions 3.18 and 3.19. The proof of the Proposition 3.18 is a straightforward generalisation of the one presented for the two examples Ia and Ib in the Lemma B.1. The only change lies in the extraction of the degrees of freedom, which impacts the matrix only on the top left two by two blocks describing the coordinate-wise behaviours. As the extraction fulfils the Assumption 3.12, the rows involving terms whose projection onto the kernel can be expressed from a same monomial are linearly independent. Thus, the same arguments as above can be applied and the conclusion follows.

In particular, by this admissibility criterion there cannot be more than $d+1$ polynomials reducing to the same moments' kernel or to an equivalent point-value quantifier. Thus, as we have $d+1$ coordinate-wise moments to tune per decomposed monomial, there is no over-determination at a fixed polynomial degree. The constraint on the extraction of degrees of freedom ensures the non over-determination overall. Further, the linear independence of the sub-matrix's columns is ensured as those polynomials cannot be linearly dependent. Thus, by linearity of the degrees of freedom, the independence of the kernels transfers to the moments and there is no row dependency. The submatrix block corresponding to any specific order is therefore invertible, and the same conclusion as in the proof B.1 follows.

The Proposition 3.18 thus holds by the number of degrees of freedom, matching the dimension of the space $\mathbb{H}_{k}(K)$. Indeed, as by the Proposition 3.19 the kernel of the linear operator defined by the set of degrees of freedom has a null kernel providing their unisolvence when enclosed within the space $\mathbb{H}_{k}(K)$.

Proof of the relation (3.4). As for any $l_{1} \leq 0$ the two natural subspaces are in direct sum, recalling the block construction of $\mathbb{H}_{k}(K)$ allows the dimension of the space $\mathbb{H}_{k}(K)$ to be easily derived. We can simply add the dimension of the two main subspaces $\left(A_{k}\right)^{d}$ and $x B_{k}$ to retrieve the dimension of $\mathbb{H}_{k}(K)$. Let us derive their respective dimensions.

First, we compute the dimension of $A_{k}$. In the way presented in [11], one can get it by using the superposition theorem. Indeed, for any second member belonging to $\mathbb{Q}_{m_{1}}$ and any boundary function $p_{k} \mathbb{1}_{f} \in L^{2}(K)$, there exists a unique solution to the Poisson's problems defining $A_{k}$ (see e.g. [8]). Thus, reading out the structure of the set $A_{k}$ implies the following relation.

$$
\begin{aligned}
\operatorname{dim} A_{k} & =\operatorname{dim} \mathcal{H}_{l_{1}}(\partial K)+\operatorname{dim} \mathbb{Q}_{m_{1}}(K) \\
& =n\left(l_{1}+1\right)^{d-1}+\left(m_{1}+1\right)^{d} .
\end{aligned}
$$

Therefore, as $\left(A_{k}\right)^{d}$ is a simple Cartesian product of $d$ copies of $A_{k}$, we have immediately $\operatorname{dim} A_{k}=d\left(\operatorname{dim} A_{k}\right)=$ $d\left(n\left(l_{1}+1\right)^{d-1}+\left(m_{1}+1\right)^{d}\right)$. In the exact same way, we retrieve the dimension of $B_{k}$ by

$$
\begin{aligned}
\operatorname{dim} B_{k} & =\operatorname{dim} \mathcal{H}_{l_{2}}(\partial K)+\operatorname{dim} \mathbb{Q}_{\left[m_{2}\right]}(K) \\
& =n\left(l_{2}+1\right)^{d-1}+\left(m_{2}+1\right)^{d}-m_{2}^{d} .
\end{aligned}
$$

Last, we recall that the space $x B_{k}$ simply corresponds to an identical $d$-duplication of the space $B_{k}$ where each coordinate has been multiplied by the corresponding spatial variable. Thus, there is no liberty adjunction during its construction, and the dimension of $x B_{k}$ equals the one of $B_{k}$. By combining this, we finally get

$$
\begin{aligned}
\operatorname{dim} \mathbb{H}_{k}(K) & =d \operatorname{dim} A_{k}+\operatorname{dim} B_{k} \\
& =d\left(n\left(l_{1}+1\right)^{d-1}+\left(m_{1}+1\right)^{d}\right)+n\left(l_{2}+1\right)^{d-1}+\left(m_{2}+1\right)^{d}-m_{2}^{d} .
\end{aligned}
$$

Finally, we point out the relation between the proposed spaces and the slight restriction of the one introduced in [12]. Indeed, we focus on relation (3.19).

Sketch of the proof that $\tilde{\mathcal{V}}_{2, k}(K) \subset \mathbb{H}_{k}(K)$. Let us quickly show that any element of $\tilde{\mathcal{V}}_{2, k}(K)$ can be recast as an element of $\mathbb{H}_{k}(K)$ for the coefficients $\left(l_{1}, l_{2}\right)=(0, k)$ and $\left(m_{1}, m_{2}\right)=(k-1,-1)$.

Regularity. Any element $v \in \tilde{\mathcal{V}}_{2, k}(K)$ belongs to $H^{1}(K)$. Indeed, $v \in H(\operatorname{div}, K) \cap H(\operatorname{rot}, K) \subset\left(H^{1}(K)\right)^{d}$. Furthermore, as any element $w$ of $\mathbb{H}_{k}(K)$ writes $w=u_{1}+x u_{2}$ with $u_{1}, u_{2}$ both having for regularity $H^{1}(K)$, 
$w \in H_{l o c}^{1}(K)$. And since $K$ is compact and bounded, $H_{l o c}^{1}(K)=H^{1}(K)$. Thus, the regularity asked for any element $v$ to be in $\tilde{\mathcal{V}}_{2, k}(K)$ is stricter than the one asked for any element $w$ to be in $\mathbb{H}_{k}(K)$.

On the boundary. Any $v \in \tilde{\mathcal{V}}_{2, k}(K)$ is a polynomial and satisfies $\left.v \cdot n\right|_{f} \in \mathbb{P}_{f}(K)$ on every face $K$ of the polygon $K$. But $\left.v \cdot n\right|_{f}$ is nothing else than the linear combination $\sum_{i=1}^{d} v_{i} n_{x_{i}}$ of the coordinate-wise functions $v_{i}$ with the normal's coefficients $n_{x_{i}}$. Thus, each polynomial $v_{i}$ has no choice but to live in the space $\mathbb{P}_{k, \ldots, k, k+1, k, \ldots, k}(K)$, where the $k+1$ is in the $i$ th position. The space $\times_{i=1}^{d} \mathbb{P}_{k, \ldots, k, k+1, k, \ldots, k}(K)$ is indeed the smallest space that is polynomial (required by the linear combination) and that contains all the functions $v$ such that $\left.v \cdot n\right|_{f} \in \mathbb{P}_{f}(K)$. Note that allowing a higher degree in the $i$ th variable is required as on each face $f \in \partial K, x \cdot n \equiv c$ for some constant $c$. And $\times_{i=1}^{d} \mathbb{P}_{k, \ldots, k, k+1, k, \ldots, k}(K) \subset \mathbb{P}_{0}(K)+x \mathbb{P}_{k}(K)$, which is exactly the structure of the $\mathbb{H}_{k}(K)$ space on the boundary.

Within the element. For any $v \in \tilde{\mathcal{V}}_{2, k}(K)$, it holds

$$
\begin{cases}\nabla(\nabla \cdot v) & \in \nabla\left(\mathbb{P}_{k-1}(K)\right) \\ \nabla \times v & \in \mathbb{P}_{k-1}(K)\end{cases}
$$

Thus, it comes $\underbrace{\nabla(\nabla \cdot v)}_{\in \nabla\left(\mathbb{P}_{k-1}(K)\right)}-\underbrace{\nabla \times(\nabla \times v)}_{\in \nabla\left(\mathbb{P}_{k-2}(K)\right)} \in \nabla\left(\mathbb{P}_{k-1}(K)\right)$, which implies $\nabla^{2} v \in \nabla\left(\mathbb{P}_{k-1}(K)\right)$ and writes

$$
\nabla^{2} v=\left(\begin{array}{c}
\sum_{i=1}^{d} \frac{\partial^{2} v_{1}}{\partial x_{i}^{2}} \\
\vdots \\
\sum_{i=1}^{d} \frac{\partial^{2} v_{d}}{\partial x_{i}^{2}}
\end{array}\right) \in\left(\begin{array}{c}
\mathbb{P}_{k-2, k-1, \ldots, k-1}(K) \\
\vdots \\
\mathbb{P}_{k-1, k-1, \ldots, k-2}(K)
\end{array}\right)
$$

Thus, we have naturally

$$
\nabla^{2} v \in\left\{u,\left(\begin{array}{c}
\Delta u_{1} \\
\vdots \\
\Delta u_{d}
\end{array}\right), \Delta u_{i} \in \mathbb{Q}_{k-1}(K)\right\}
$$

Summary. Let $v \in \mathcal{V}_{2, k}(K)$. Then $v$ can be decomposed as follows:

$$
v=\left.\left(\dot{v}_{1}+\dot{\circ}_{2}\right)\right|_{K}+\left.\left(\overline{v_{1}}+\overline{v_{2}}\right)\right|_{\partial K}
$$

where

$$
\begin{cases}\overline{v_{1}} \in\left(\mathbb{P}_{0}(\partial K)\right)^{d} & \text { represents the constant part of } v \text { on the boundary } \\ \overline{v_{2}} \in\left(\mathbb{P}_{k}(\partial K) \backslash \mathbb{P}_{0}(\partial K)\right)^{d} & \text { represents the higher parts of } v \text { on the boundary }\end{cases}
$$

and

$$
\left\{\begin{array}{l}
v_{1}^{\circ}=\left.v\right|_{K}-p_{[1], \ldots,[1]} \\
\overline{v_{2}}=p_{[1], \ldots,[1]}
\end{array}\right.
$$

for any $p_{[1], \ldots,[1]}$ belonging to $x \mathbb{P}_{0}$. Doing so, we get

and

$$
\begin{cases}\left.\bar{v}_{1}\right|_{\partial K} & \in\left(\mathbb{P}_{0}(\partial K)\right)^{d} \\ \left.\dot{\circ}_{1}\right|_{K} & =\left.v\right|_{K}-p_{[1], \ldots,[1]}\end{cases}
$$

$$
\begin{cases}\left.\overline{v_{2}}\right|_{\partial K} & \left(\mathbb{P}_{k}(\partial K) \backslash \mathbb{P}_{0}(\partial K)\right)^{d} \\ \left.\wp_{2}\right|_{K} & =p_{[1], \ldots,[1]}\end{cases}
$$


There, we get straightforwardly from the previous paragraphs:

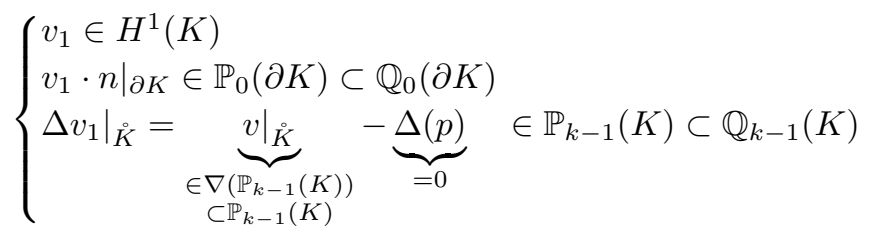

and

$$
\begin{cases}v_{2} \in H^{1}(K) & \\ \left.v_{2} \cdot n\right|_{\partial K}=\left.v\right|_{\partial K} \cdot n-\left.v_{1} \cdot n\right|_{\partial K} & \in \mathbb{P}_{k}(\partial K) \subset \mathbb{Q}_{k}(\partial K) \\ \left.\Delta v_{2}\right|_{K} ^{\circ}=\Delta(x c)=x \Delta(c)=0 & \in \mathbb{P}_{[-1]}(K) \subset \mathbb{Q}_{[-1]}(K)\end{cases}
$$

for some constant $c \in \mathbb{P}_{0}(K)$. Writing $v_{2}$ as $v_{2}=x w$ on the boundary with $w \in x \mathbb{P}_{k, \ldots, k}(\partial K)$ $\subset \times_{i=1}^{d} \mathbb{P}_{k, \ldots, k, k+1, k, \ldots, k}(\partial K) \backslash \mathbb{P}_{0}(\partial K)$, it comes further

$$
\begin{cases}w \in H^{1}(K) & \\ \left.w \cdot n\right|_{\partial K} & \in \mathbb{P}_{k}(\partial K) \subset \mathbb{Q}_{k}(\partial K) \\ \left.\Delta w\right|_{K}=0 & \in \mathbb{P}_{-1}(K) \subset \mathbb{Q}_{[-1]}(K) .\end{cases}
$$

Taking without loss of generality $c=1$, considering $v=v_{1}+x w$ and setting $\left(l_{1}, l_{2}\right)=(0, k),\left(m_{1}, m_{2}\right)=$ $(k-1,-1)$, we get:

$$
v_{1} \in \mathcal{A}_{k}, \quad v_{2}=x w \in x \mathcal{B}_{k},
$$

and therefore,

$$
v=v_{1}+v_{2} \in \mathbb{H}_{k}(K)
$$

Acknowledgements. E. Le Mélédo and P. Öffner have been funded by SNF project 200020_175784 "Solving advection dominated problems with high order schemes with polygonal meshes: application to compressible and incompressible flow problems". P. Öffner has also been funded by an University of Zürich Forschungskredit grant. The constructive criticisms of the three referees are also acknowledged.

\section{REFERENCES}

[1] R. Abgrall, E. Le Mélédo and P. Öffner, On the connection between residual distribution schemes and flux reconstruction. Preprint: 1807.01261 (2018).

[2] R. Abgrall, É. Le Mélédo and P. Öffner, A class of finite dimensional spaces and $H$-(div) conformal elements on general polytopes. Preprint: 1907.08678 (2019).

[3] J. Aghili, D. Di Pietro and B. Ruffini, An $h p$-hybrid high-order method for variable diffusion on general meshes. Comput. Methods Appl. Math. 17 (2017) 359-376.

[4] L. Beirão da Veiga, F. Brezzi, L.D. Marini and A. Russo, Mixed virtual element methods for general second order elliptic problems on polygonal meshes. ESAIM:M2AN 50 (2016) 727-747.

[5] F. Bonaldi, D. Di Pietro, G. Geymonat and F. Krasucki, A hybrid high-order method for kirchhoff-love plate bending problems. ESAIM:M2AN 52 (2018) 393-421.

[6] L. Botti, D. Di Pietro, and J. Droniou, A hybrid high-order method for the incompressible navier-stokes equations based on temam's device. J. Comput. Phys. 376 (2019) 786-816.

[7] F. Brezzi, J. Douglas and L.D. Marini, Two families of mixed finite elements for second order elliptic problems. Numer. Math. 47 (1985) 217-235.

[8] J. Chabrowski, The Dirichlet Problem with L2-boundary Data for Elliptic Linear Equations. Springer (2006).

[9] W. Chen and Y. Wang, Minimal degree $H$ (curl) and $H$ (div) conforming finite elements on polytopal meshes. Math. Comput. 86 (2017) 2053-2087.

[10] B. Cockburn, G.E. Karniadakis, and C.W. Shu, Discontinuous Galerkin Methods: Theory, Computation and Applications. Springer Science \& Business Media 11 (2012).

[11] L.B. Da Veiga, F. Brezzi, A. Cangiani, G. Manzini, L.D. Marini and A. Russo, Basic principles of virtual element methods. Math. Models Methods Appl. Sci. 23 (2013) 199-214. 
[12] L.B. Da Veiga, F. Brezzi, L.D. Marini and A. Russo, H(div) and H(curl)-conforming virtual element methods. Numer. Math. 133 (2016) 303-332.

[13] L.B. Da Veiga, F. Brezzi, L.D. Marini and A. Russo, Mixed virtual element methods for general second order elliptic problems on polygonal meshes. ESAIM: M2AN 50 (2016) 727-747.

[14] D. Di Pietro and S. Lemaire, An extension of the Crouzeix-Raviart space to general meshes with application to quasiincompressible linear elasticity and stokes flow. Math. Comput. 84 (2015) 1-31.

[15] D.A. Di Pietro and A. Ern, Arbitrary-order mixed methods for heterogeneous anisotropic diffusion on general meshes. IMA J. Numer. Anal. 37 (2016) 40-63.

[16] F. Dubois, I. Greff and C. Pierre, Raviart-Thomas finite elements of petrov-galerkin type. ESAIM:M2AN 53 (2017) 1553-1576.

[17] A. Gillette, A. Rand and C. Bajaj, Construction of scalar and vector finite element families on polygonal and polyhedral meshes. Comput. Methods Appl. Math. 16 (2016) 667-683.

[18] H.T. Huynh, A flux reconstruction approach to high-order schemes including discontinuous galerkin methods. In: 18th AIAA Computational Fluid Dynamics Conference (2007) 4079.

[19] P. Lesaint and P. Raviart, On a finite element method for solving the neutron transport equation, edited by C. De Boor. In: Mathematical Aspects of Finite Element in Partial Differential Equations. Academic Press (1974) 89-123.

[20] K. Lipnikov, G. Manzini and M. Shashkov, Mimetic finite difference method. J. Comput. Phys. 257 (2014) $1163-1227$.

[21] R. Loubère, P.H. Maire and M. Shashkov, Reale: a reconnection arbitrary-lagrangian-eulerian method in cylindrical geometry. Comput. Fluids 46 (2011) 59-69.

[22] J. Nédélec, Mixed finite elements in $\mathbb{R}^{3}$. Numer. Math. 35 (1980) 315-341.

[23] H. Ranocha, P. Öffner and T. Sonar, Summation-by-parts operators for correction procedure via reconstruction. J. Comput. Phys. 311 (2016) 299-328.

[24] P.A. Raviart and J.M. Thomas, A mixed finite element method for 2-nd order elliptic problems. In: Mathematical Aspects of Finite Element Methods. Springer Berlin Heidelberg, Berlin, Heidelberg (1977) 292-315.

[25] W. Reeds and T. Hill, Triangular mesh methods for the neutron transport equation. Technical Report LA-UR-73-479. Los Alamos (1973).

[26] N. Sukumar and E.A. Malsch, Recent advances in the construction of polygonal finite element interpolants. Arch. Comput. Methods Eng. 13 (2006) 129.

[27] C. Talischi, A. Pereira, G.H. Paulino, I.F.M. Menezes and M.S. Carvalho, Polygonal finite elements for incompressible fluid flow. Int. J. Numer. Methods Fluids 74 (2013) 134-151.

[28] P. Vincent, P. Castonguay and A. Jameson, A new class of high-order energy stable flux reconstruction schemes. J. Sci. Comput. 47 (2011) 50-72. 